\title{
The N-Point Definite Integral Approximation Formula (N-POINT DIAF)
}

\author{
Francis Oketch Ochieng', Nicholas Muthama Mutua ${ }^{*}$, Nicholas Mwilu Mutothya
}

Mathematics and Informatics Department, Taita Taveta University, Voi, Kenya

\section{Email address:}

nicholasmuthama.mutua@gmail.com (N. M. Mutua), oketch.francis@yahoo.com (F. O. Ochieng'), nicholasmuthama@ttu.ac.ke (N. M. Mutua),nichmwilu@gmail.com (N. M. Mutothya)

${ }^{*}$ Corresponding author

\section{To cite this article:}

Francis Oketch Ochieng', Nicholas Muthama Mutua, Nicholas Mwilu Mutothya. The N-Point Definite Integral Approximation Formula (NPOINT DIAF). Applied and Computational Mathematics. Vol. 6, No. 1, 2017, pp. 1-33. doi: 10.11648/j.acm.20170601.11

Received: November 20, 2016; Accepted: January 24, 2017; Published: February 21, 2017

\begin{abstract}
Various authors have discovered formulae for numerical integration approximation. However these formulae always result to some amount of error which may differ in size depending on the formula. It's therefore important that a formula with highest precision has been discovered and should be implemented for use in numerical integration approximations problems, especially for the definite integrals which cannot be evaluated by applying the analytical techniques. The present paper therefore explores the derivation of the N-point Definite Integral Approximation Formula (N-point DIAF) which amounts to the discovery of the 2-Point DIAF. This formula will assist in almost accurate evaluation of all definite integrals numerically. The proof of the formula is given, a specific test problem is then solved using the discovered 2-Point DIAF to obtain the solution numerically, which has the highest precision compared to other numerical methods of integration. Further the error terms are obtained and compared with the existing methods. Finally, the effectiveness of the proposed formula is illustrated by means of a numerical example.
\end{abstract}

Keywords: Numerical Integration, Approximation, Definite Integrals, Error, Analytical Techniques, Stability

\section{Introduction}

Integrals of most analytical functions can be evaluated as

$$
I=\int_{a}^{b} f(x) d x=F(b)-F(a)
$$

where $F(x)$ is differentiable function whose derivative is $f(x)$ i.e.

$$
F^{\prime}(x)=f(x)
$$

Often need arises for evaluating the definite integral of functions that does not have explicit antiderivative, in other circumstances the function is not known explicitly but is given empirically by a set of measured or tabulated values.

In circumstances where the integral cannot be evaluated analytically, numerical integration is used to give approximate solution to the definite integral. Since solutions obtained in numerical integration are approximate, there is usually error of approximation which is a measure of the deviation of the approximate solution from the exact value.

Thus the best solution is that which converges to the exact solution.

In calculus and engineering mathematics courses, we learnt many methods to solve the integral problems, including change of variables method, integration by parts method, partial fractions method, trigonometric substitution method, and so on [2]. In this paper, we present a formula to approximate definite integrals.

\section{Objective}

To approximate the definite integral $\int_{a}^{b} w(x) f(x) d x$, where the weight function, $w(x)>0$ in a closed interval $[a, b]$ using the newly developed N-point Definite Integral Approximation Formula (N-point DIAF) and discuss the 
accuracy, convergence and stability of the method. Here, $a$ and $b$ are the limits of integration.

\section{Literature Review}

[4] notes that Numerical integration is the study of how the numerical value of an integral can be found. Also called quadrature, which refers to finding a square whose area is the same as the area under a curve, it is one of the classical topics of numerical analysis. Of central interest is the process of approximating a definite integral from values of the integrand when exact mathematical integration is not available. Many methods are available for approximating the integral to the desired precision in Numerical integration. A new set of numerical integration formula of Open Newton-Cotes Quadrature with Midpoint Derivative type is suggested, which is the modified form of Open Newton-Cotes Quadrature [9]. Numerical integration is the process of computing the value of a definite integral from a set of numerical values of the integrand. The process of evaluation of integration of a function of a single variable is sometimes called Mechanical Quadrature. The computation of a double integral of a function of two independent variables is called Mechanical Cubature. There are many methods are available for numerical integration [8].

Integral Calculus is a fundamental field of study in Mathematics and is widely used to model physical processes by scientists and engineers [3]. It has widespread uses in science, engineering and economics and can solve many problems that Algebra alone cannot [1]. Most of the models are always definite integrals which need to be evaluated in order to get a solution that provide a basis for drawing a conclusion. Most of the integrals are easy to evaluate using analytical methods. However, some integrals cannot be evaluated analytically and therefore need to be approximated. This means that we have to apply numerical methods in order to get an approximate solution. This is referred to as numerical integration.

Several authors have discovered formulae for approximating such integrals which can only be evaluated numerically. [5] reiterate that some of the great mathematicians and scientists such as Romberg, Simpson, Gauss-Legendre, Gauss-Chebyshev, Newton-Cotes, for instance, have made remarkable contribution in this area. Sir Isaac Newton made use of forward difference operator and forward difference table to simplify the calculations involved in the polynomial approximation of functions which are known at equally spaced data points. Thus, the Newton Forward Difference Interpolating Polynomial (NFDIP) of degree 4 provides a basic foundation upon which the N-point DIAF is based [6]. It is worthwhile to note further that the choice of the variables used in the N-point DIAF, stated in the next section below, does not in any way suggest any correlation with the neighborhood method used in Regression Analysis (i.e. the k-Nearest Neighbours Regression algorithm).

\subsection{Statement of the Formula}

Suppose $f(x)$ is a function of the equally spaced argument $\mathrm{x}$, which may be given explicitly or as a tabulated data. Then we evaluate definite integral in closed interval [a, b] as $I=\int_{a}^{b} f(x) d x$ we can define the integral $\int_{a}^{b} w(x) f(x) d x$ where $w(x)$ is the weight function. Let $h=\frac{b-a}{4 N}$, where $\mathrm{h}$ is the length the interval $\left[x_{i}, x_{i+1}\right]$ and $\mathrm{N}$ is the number of sub-intervals and $w(x)=1$ then the N-point DIAF for $\int_{a}^{b} w(x) f(x) d x$ is given by:

$$
\int_{a}^{b} w(x) f(x) d x=\frac{2 h}{45} \sum_{k=0}^{4 N} \lambda_{k} f(a+k h)
$$

where the weights are given by

$$
\lambda_{k}=\left\{\begin{array}{l}
7, \text { if }(k=\{0,4 N\}) \\
y, \text { otherwise }
\end{array}\right.
$$

where

$$
N=1,2,3, \ldots, y=\left[32+(-1)^{k-1}\left(\frac{\left(2^{k}+(-2)^{k}\right)}{\left\{\frac{r}{2} \frac{2^{k-1}}{5}+\left(1-\frac{r}{2}\right) \frac{2^{k}}{9}\right\}}\right)\right]
$$

and $r=k \bmod 4$ (that is $r$ is the remainder when $k$ is divided by 4 ).

It is important to note that the proof of this formula is explored in the next section below, and it explains the genesis behind it in detail.

\subsection{Formula Proof}

Let $h=\frac{b-a}{4 N}$ and $w(x)=1$, then to approximate the definite integral $\int_{a}^{b} w(x) f(x) d x$ :

We subdivide the interval $[a, b]$ into $4 N$ subdivisions of equal width $h$ and then fit a polynomial of degree 4 on $\left[x_{0}, x_{4}\right],\left[x_{4}, x_{8}\right],\left[x_{8}, x_{12}\right], \ldots,\left[x_{4 N-4}, x_{4 N}\right]$. Here, $x_{0}, x_{1}, x_{2}, x_{3}, \ldots, x_{4 N-1}, x_{4 N}$ are suitable points in the interval of integration.

Using the NFDIP (Newton Forward Difference Interpolating Polynomial), we approximate 


$$
\begin{aligned}
f(x)= & f_{0}+s \Delta f_{0}+\frac{s(s-1)}{2 !} \Delta^{2} f_{0}+\frac{s(s-1)(s-2)}{3 !} \Delta^{3} f_{0} \\
& +\frac{s(s-1)(s-2)(s-3)}{4 !} \Delta^{4} f_{0}+R_{N}
\end{aligned}
$$

Neglecting the error term, $R_{N}$, as it is too small hence negligible, we then approximate $\int_{a}^{b} w(x) f(x) d x$ in each 4 subdivisions and then summing them up as follows:

where $R_{N}$ is the error term, $s=\frac{x-a}{h}$, and $\Delta$ is the forward difference operator (i.e. $\Delta f_{k}=f_{k+1}-f_{k}$, by definition).

$$
\begin{aligned}
& \int_{a}^{b} f(x) d x \approx I_{1}+I_{2}+I_{3}+\ldots+I_{N} \approx I_{1}+I_{2}+I_{3}+\ldots+I_{N} \\
& \approx \int_{x_{0}}^{x_{4}} f(x) d x+\int_{x_{4}}^{x_{8}} f(x) d x+\int_{x_{8}}^{x_{12}} f(x) d x+\ldots \\
& +\int_{x_{4 N-4}}^{x} f(x) d x \\
& \Rightarrow I_{1}=\int_{x_{0}}^{x_{4}} f(x) d x \approx h \int_{0}^{4}\left(\begin{array}{l}
f_{0}+s \Delta f_{0}+\frac{s(s-1)}{2 !} \Delta^{2} f_{0}+ \\
\frac{s(s-1)(s-2)}{3 !} \Delta^{3} f_{0} \\
+\frac{s(s-1)(s-2)(s-3)}{4 !} \Delta^{4} f_{0}
\end{array}\right) d s,\left(\text { since, } s=\frac{x-a}{h} \Rightarrow d s=\frac{1}{h} d x, x_{0}=a\right) \\
& \Rightarrow I_{1} \approx h \int_{0}^{4}\left(\begin{array}{l}
f_{0}+s \Delta f_{0}+\frac{\left(s^{2}-s\right)}{2} \Delta^{2} f_{0}+\frac{\left(s^{3}-3 s^{2}+2 s\right)}{6} \Delta^{3} f_{0} \\
+\frac{\left(s^{4}-6 s^{3}+11 s^{2}-6 s\right)}{24} \Delta^{4} f_{0}
\end{array}\right) d s \\
& \Rightarrow I_{1} \approx h\left[\begin{array}{l}
s f_{0}+\frac{s^{2}}{2} \Delta f_{0}+\frac{\left(\frac{s^{3}}{3}-\frac{s^{2}}{2}\right)}{2} \Delta^{2} f_{0}+\frac{\left(\frac{s^{4}}{4}-s^{3}+s^{2}\right)}{6} \Delta^{3} f_{0} \\
+\frac{\left(\frac{s^{5}}{5}-\frac{3}{2} s^{4}+\frac{11}{3} s^{3}-3 s^{2}\right)}{24} \Delta^{4} f_{0}
\end{array}\right] 0 \\
& \Rightarrow I_{1} \approx h\left[4 f_{0}+8 \Delta f_{0}+\frac{20}{3} \Delta^{2} f_{0}+\frac{8}{3} \Delta^{3} f_{0}+\frac{14}{45} \Delta^{4} f_{0}\right] \\
& \Rightarrow I_{1} \approx h\left[\begin{array}{l}
4 f_{0}+8\left(f_{1}-f_{0}\right)+\frac{20}{3}\left(f_{2}-2 f_{1}+f_{0}\right)+\frac{8}{3}\left(f_{3}-3 f_{2}+3 f_{1}-f_{0}\right) \\
+\frac{14}{45}\left(f_{4}-4 f_{3}+6 f_{2}-4 f_{1}+f_{0}\right)
\end{array}\right] \\
& \left.\Rightarrow I_{1} \approx h\left[\frac{14}{45} f_{0}+\frac{64}{45} f_{1}+\frac{24}{45} f_{2}+\frac{64}{45} f_{3}+\frac{14}{45} f_{4}\right)\right] \\
& \left.\Rightarrow I_{1} \approx \frac{2 h}{45}\left[7 f_{0}+32 f_{1}+12 f_{2}+32 f_{3}+7 f_{4}\right)\right]
\end{aligned}
$$

Similarly, $\left.\left.\quad I_{2} \approx \frac{2 h}{45}\left[7 f_{4}+32 f_{5}+12 f_{6}+32 f_{7}+7 f_{8}\right)\right] \quad I_{3} \approx \frac{2 h}{45}\left[7 f_{8}+32 f_{9}+12 f_{10}+32 f_{11}+7 f_{12}\right)\right]$ 
$I_{N} \approx \frac{2 h}{45}\left[\begin{array}{l}7 f_{4 N-4}+32 f_{4 N-3}+12 f_{4 N-2} \\ \left.+32 f_{4 N-1}+7 f_{4 N}\right)\end{array}\right]$

Thus,

$$
\int_{a}^{b} f(x) d x \approx \frac{2 h}{45}\left[\begin{array}{l}
7\left(f_{0}+f_{4 N}\right)+12\left(f_{2}+f_{6}+f_{10}+\ldots+f_{4 N-2}\right) \\
+32\left(f_{1}+f_{3}+f_{5}+f_{7}+\ldots+f_{4 N-3}+f_{4 N-1}\right) \\
+14\left(f_{4}+f_{8}+\ldots+f_{4 N-4}\right)
\end{array}\right]
$$

$\Rightarrow \int_{a}^{b} w(x) f(x) d x=\frac{2 h}{45} \sum_{k=0}^{4 N} \lambda_{k} f_{k}, \quad$ but $f_{k}=f\left(x_{k}\right)$ and $x_{k}=\left(x_{o}+k h\right)=(a+k h)$. Therefore,

$$
\Rightarrow \int_{a}^{b} w(x) f(x) d x=\frac{2 h}{45} \sum_{k=0}^{4 N} \lambda_{k} f(a+k h)
$$

which conforms to the statement of the N-point DIAF so long as we can find the values for the weights, $\lambda_{k}$ 's 's.

Now, from equation (6) it can be clearly seen that the weights $\lambda_{k}=7$ whenever $k=0, k=4 N$; Or $\lambda_{k}=y$ whenever $1 \leq k \leq 4 N-1 ; y$ is dependent on $k$, i.e. $\lambda_{k}=\left\{\begin{array}{l}7, \text { if }(k=\{0,4 N\}) \\ y, \text { if } 1 \leq k \leq 4 N-1\end{array}\right.$, where the values of the multiplier, $y$, oscillate back and forth between $12 \longleftrightarrow 32 \longleftrightarrow 14$ starting counting from $k=1$ at 32 then Left-Right manner in steps of 1 respectively until the last count reaches $k=4 N-1$. [Here, $k=1(1) 4 N-1$ ]. This means that

$$
\left.\begin{array}{l}
\lambda_{0}=7, \lambda_{1}=32, \lambda_{2}=12, \lambda_{3}=32, \\
\lambda_{4}=14, \ldots, \lambda_{4 N-4}=14, \lambda_{4 N-3}=32, \\
\lambda_{4 N-2}=12, \lambda_{4 N-1}=32
\end{array}\right\}
$$

Our main task is to find the expression for $y$ as follows.

If we subtract each $\lambda_{k}$ value from 32 [i.e. $\left(32-\lambda_{k}\right)$ ], then it follows that

$$
\lambda_{k}=y=32+(-1)^{k}\left(\frac{a_{k}}{b_{k}}\right)
$$

where $a_{k}$ and $b_{k}$ depend on $k$.

The values of $\left(\frac{a_{k}}{b_{k}}\right)$ now oscillate back and forth between $20 \longleftrightarrow 0 \longleftrightarrow 18$

[i.e, $\quad(32-12) \longleftrightarrow(32-32) \longleftrightarrow(32-14) \quad$ ] starting counting from $k=1$ at 0 then Left-Right manner in steps of 1

\begin{tabular}{|c|c|}
\hline$k$ & $\left(\frac{a_{k}}{b_{k}}\right)$ \\
\hline 1 & $\frac{2^{1}+(-2)^{1}}{1}=0$ \\
\hline 2 & $\frac{2^{2}+(-2)^{2}}{\frac{2^{1}}{5}}=20$ \\
\hline 3 & $\frac{2^{3}+(-2)^{3}}{1}=0$ \\
\hline 4 & $\frac{2^{4}+(-2)^{4}}{\frac{2^{4}}{9}}=18$ \\
\hline 5 & $\frac{2^{5}+(-2)^{5}}{1}=0$ \\
\hline 6 & $\frac{2^{6}+(-2)^{6}}{\frac{2^{5}}{5}}=20$ \\
\hline 7 & $\frac{2^{7}+(-2)^{7}}{1}=0$ \\
\hline 8 & $\frac{2^{8}+(-2)^{8}}{\frac{2^{8}}{9}}=18$ \\
\hline . & . \\
\hline $4 N-4$ & $\frac{2^{4 N-4}+(-2)^{4 N-4}}{\frac{2^{4 N-4}}{9}}=18$ \\
\hline $4 N-3$ & $\frac{2^{4 N-3}+(-2)^{4 N-3}}{1}=0$ \\
\hline $4 N-2$ & $\frac{2^{4 N-2}+(-2)^{4 N-2}}{\frac{2^{4 N-3}}{5}}=20$ \\
\hline $4 N-1$ & $\frac{2^{4 N-1}+(-2)^{4 N-1}}{1}=0$ \\
\hline
\end{tabular}
respectively until the last count reaches $k=4 N-1$ [Here, $k=1(1) 4 N-1$ ], to yield the same values of $\lambda_{k}$ 's 's as in (8) above. This means that we can compile a table for the values of $k$ against $\left(\frac{a_{k}}{b_{k}}\right)$ for equation (9) as shown in Table 1 below Table 1. Values of $k$ against $\left(\frac{a_{k}}{b_{k}}\right)$ for equation (9a). 
From the Table 1.0 above, it's evident that

$$
a_{k}=2^{k}+(-2)^{k}
$$

and the values of $b_{k}$ form the sequence:

$$
\left.\begin{array}{l}
1, \frac{2^{1}}{5}, 1, \frac{2^{4}}{9}, 1, \frac{2^{5}}{5}, 1, \frac{2^{8}}{9}, \ldots, 1 \\
\left.p \frac{2^{k-1}}{5}+q \frac{2^{k}}{9}\right)+, \ldots,+1
\end{array}\right\}
$$

$p$ and $q$ take values 0 and 1 alternating [i.e. when $p=0$, $q=1$ and when $p=1, q=0]$.

Thus the $k^{\text {th }}$ term, $b_{k}$, is given by

$$
b_{k}=\left\{\begin{array}{l}
p \frac{2^{k-1}}{5}+q \frac{2^{k}}{9}, \text { if }(k \bmod 2=0) \\
1, \quad \text { otherwise }
\end{array}\right.
$$

where $p$ and $q$ are dependent on $k$ and are to be evaluated as follows

If we now let $r=k \bmod 4$ (i.e. $r$ is the remainder when $k$ is divided by 4 ), then it follows that when $r=2 \Rightarrow p=1$ and $q=0$, so that $b_{k}=\frac{2^{k-1}}{5}$, which satisfy the sequence (11) above.

Also when $r=0 \Rightarrow p=0$ and $q=1$ so that $b_{k}=\frac{2^{k}}{9}$, which also satisfy the sequence (11) above.

Thus, there is a linear relationship between $r$ and $p$ which transforms the discrete extreme points $[1,0]$ in $p$ onto $[2,0]$ in $r$, i.e

$$
[1,0] \rightarrow[2,0]
$$

If we let this relationship to be

$$
p=m_{1} r+c_{1}
$$

where $m_{1}$ and $c_{1}$ are constants to be evaluated for relationship (12) to hold.

Plugging the values of (12) into (13) yields

$$
p=\frac{r}{2}
$$

This is because,

$$
2=1 m_{1}+c_{1}
$$

and

$$
0=0 m_{1}+c_{1}
$$

Solving (14a) and (14b) simultaneously yields (14).

Similarly, there is a linear relationship between $r$ and $q$ which transforms the discrete extreme points $[1,0]$ in $q$ onto $[2,0]$ in $r$, i.e.

$$
[0,1] \rightarrow[2,0]
$$

If we let this relationship to be

$$
q=m_{2} r+c_{2}
$$

where $m_{2}$ and $c_{2}$ are constants to be evaluated for relationship (15) to hold.

Plugging the values of (10) into (11) yields

$$
q=\left(1-\frac{r}{2}\right)
$$

This is because,

$$
2=0 m_{2}+c_{2}
$$

and

$$
0=1 m_{2}+c_{2}
$$

Solving (17a) and (17b) simultaneously yields (17)

Substituting equations (14) and (17) into equation (11) and neglecting all the values for $b_{k}=1$, as they are insignificant in the final result for $y$, yields

$$
b_{k}=\frac{r}{2} \frac{2^{k-1}}{5}+\left(1-\frac{r}{2}\right) \frac{2^{k}}{9}
$$

Substituting equation (11) and (18) into equation (9) yields

$$
y=\left[32+(-1)^{k-1}\left(\frac{\left(2^{k}+(-2)^{k}\right)}{\left\{\frac{r}{2} \frac{2^{k-1}}{5}+\left(1-\frac{r}{2}\right) \frac{2^{k}}{9}\right\}}\right)\right]
$$

as required.

\subsection{Numerical Illustration}

Approximate the integral

$\int_{0}^{1} \frac{1}{x+1} d x$; using

1) Gauss-Chebyshev 3 point formula.

2) Gauss-Legendre 3 point formula.

3) Simpson's $3 / 8$ rule with 8 subdivisions. 
4) Trapezoida rule with 1, 2, 4, and 8 subdivisions. Hence, use Romberg approximation to obtain a most accurate solution.

5) Using Boole's rule with $n=4,8$

6) Using weddles 's rule with $n=6$

7) The 2-point DIAF.

Hence calculate the absolute relative true error, $\left|\epsilon_{t}\right|$, involved in each approximation.

\section{Solution}

The exact solution of $\int_{0}^{1} \frac{1}{x+1} d x$ analytically is $[\ln (x+1)]_{0}^{1}=\ln (2)-\ln (1)=0.69314718$

Using Gauss-Chebyshev 3 point formula

$$
I=\int_{0}^{1} \frac{1}{x+1} d x
$$

We first transform the interval $[0,1]$ onto $[-1,1]$ using the transformation $x=\left(\frac{b-a}{2}\right) t+\left(\frac{b+a}{2}\right)$, where $a=0$ and $b=1 \Rightarrow x=\frac{1}{2} t+\frac{1}{2}$

$$
\left.\Rightarrow d x=\frac{1}{2} d t\right\}
$$

Substituting equation $(i i) *$ into $(i) *$ yields,

$$
I=\int_{-1}^{1} \frac{1}{3+t} d t
$$

Then by Gauss-Chebyshev 3 point formula $\frac{f(t)}{\sqrt{1-t^{2}}}=\frac{1}{3+t}$

$\Rightarrow f(t)=\frac{\sqrt{1-t^{2}}}{3+t}$

Thus,

$$
\left.\Rightarrow I=\frac{\pi}{3}\left[\frac{\sqrt{1-\left(\frac{\sqrt{3}}{2}\right)^{2}}}{3+\left(\frac{\sqrt{3}}{2}\right)}\right]+\left(\frac{\sqrt{1-0^{2}}}{3+0}\right)+\left(\frac{\sqrt{1-\left(-\frac{\sqrt{3}}{2}\right)^{2}}}{3+\left(-\frac{\sqrt{3}}{2}\right)}\right]\right]
$$

$$
=0.729864959
$$

$$
\begin{aligned}
\left|\epsilon_{t}\right| & =\left|\frac{0.69314718-0.729864959}{0.69314718}\right| \times 100 \% \\
& \approx(5.297256) \%
\end{aligned}
$$

Using Gauss-Legendre 3 point formula

$$
I=\int_{0}^{1} \frac{1}{x+1} d x
$$

We first transform the interval $[0,1]$ onto $[-1,1]$ using the transformation $x=\left(\frac{b-a}{2}\right) t+\left(\frac{b+a}{2}\right)$, where $a=0$ and

$$
\left.b=1 \Rightarrow x=\frac{1}{2} t+\frac{1}{2}, \Rightarrow d x=\frac{1}{2} d t\right\}
$$

Substituting equation $(i i) *$ into $(i) *$ yields, $I=\int_{-1}^{1} \frac{1}{3+t} d t$. $I=\int_{-1}^{1} f(t) d t=\frac{\pi}{3}\left[f\left(\frac{\sqrt{3}}{2}\right)+f(0)+f\left(-\frac{\sqrt{3}}{2}\right)\right] \quad, \quad$ here

Then by Gauss-Legendre 3 point formula $I=\int_{-1}^{1} f(t) d t=\frac{1}{9}\left[5 f\left(-\sqrt{\frac{3}{5}}\right)+8 f(0)+5 f\left(\sqrt{\frac{3}{5}}\right)\right]$, here $f(t)=\frac{1}{3+t}$.

Thus, $\Rightarrow I=\frac{1}{9}\left[5\left(\frac{1}{3-\sqrt{\frac{3}{5}}}\right)+8\left(\frac{1}{3}\right)+5\left(\frac{1}{3+\sqrt{\frac{3}{5}}}\right)\right]=0.693121693 \quad\left|\epsilon_{t}\right|=\left|\frac{0.69314718-0.693121693}{0.69314718}\right| \times 100 \%$

$\left|\frac{0.69314718-0.693121693}{0.69314718}\right| \times 100 \% \approx\left(3.6771 \times 10^{-3}\right) \%$

Using Simpson's $3 / 8$ rule with 8 subdivisions

$$
h=\frac{1-0}{8}=\frac{1}{8} ; f(x)=\frac{1}{x+1}
$$


Table 2. Values of $x$ against $f(x)=\frac{1}{x+1}$.

\begin{tabular}{llllllllll}
\hline $\boldsymbol{x}$ & $\mathbf{0}$ & $\frac{\mathbf{1}}{\mathbf{8}}$ & $\frac{\mathbf{1}}{\mathbf{4}}$ & $\frac{\mathbf{3}}{\mathbf{8}}$ & $\frac{\mathbf{1}}{\mathbf{2}}$ & $\frac{\mathbf{5}}{\mathbf{8}}$ & $\frac{\mathbf{3}}{\mathbf{4}}$ & $\frac{\mathbf{7}}{\mathbf{8}}$ & $\mathbf{1}$ \\
\hline$f(x)$ & 1 & $\frac{8}{9}$ & $\frac{4}{5}$ & $\frac{8}{11}$ & $\frac{2}{3}$ & $\frac{8}{13}$ & $\frac{4}{7}$ & $\frac{8}{15}$ & $\frac{1}{2}$ \\
\hline
\end{tabular}

Then by Sympson's 3/8 rule.

$$
\int_{a}^{b} f(x) d x=\frac{3 h}{8}\left(\begin{array}{l}
f_{0}+3\left(f_{1}+f_{2}+f_{4}+f_{5}+f_{7}+f_{8}+---+f_{3 N-2}\right. \\
\left.+f_{3 N-1}\right)+2\left(f_{3}+f_{6}+f_{9}+---+f_{3 N-3}\right)+f_{3 N}
\end{array}\right)
$$

Thus for 8 subdivisions we have,

$$
\begin{aligned}
I=\int_{0}^{1} \frac{1}{x+1} d x & =\frac{3 h}{8}\left(\begin{array}{l}
f_{0}+3\left(f_{1}+f_{2}+f_{4}+f_{5}+f_{7}\right) \\
+2\left(f_{3}+f_{6}\right)+f_{8}
\end{array}\right) \\
\Rightarrow I=\frac{3\left(\frac{1}{8}\right)}{8} & \left(\begin{array}{l}
1+3\left(\frac{8}{9}+\frac{4}{5}+\frac{2}{3}+\frac{8}{13}+\frac{8}{15}\right)+ \\
2\left(\frac{8}{11}+\frac{4}{7}\right)+\frac{1}{2}
\end{array}\right)=0.684854208 \\
\left|\epsilon_{t}\right| & =\left|\frac{0.69314718-0.684854208}{0.69314718}\right| \times 100 \% \\
& \approx 1.196423 \%
\end{aligned}
$$

Using Romberg Approximation

$$
h=\frac{1-0}{8}=\frac{1}{8} ; f(x)=\frac{1}{x+1}
$$

Table 3. Values of $x$ against $f(x)=\frac{1}{x+1}$.

\begin{tabular}{llllllllll}
\hline $\boldsymbol{x}$ & $\mathbf{0}$ & $\mathbf{1}$ & $\frac{\mathbf{1}}{\mathbf{4}}$ & $\frac{\mathbf{3}}{\mathbf{8}}$ & $\frac{\mathbf{1}}{\mathbf{2}}$ & $\frac{\mathbf{5}}{\mathbf{8}}$ & $\frac{\mathbf{3}}{\mathbf{4}}$ & $\frac{\mathbf{7}}{\mathbf{8}}$ & $\mathbf{1}$ \\
\hline$f(x)$ & 1 & $\frac{8}{9}$ & $\frac{4}{5}$ & $\frac{8}{11}$ & $\frac{2}{3}$ & $\frac{8}{13}$ & $\frac{4}{7}$ & $\frac{8}{15}$ & $\frac{1}{2}$ \\
\hline
\end{tabular}

Using trapezoidal rule with 1 subdivision.

$$
\begin{gathered}
h=\frac{1-0}{1}=1 \\
T_{1}=\int_{0}^{1} \frac{1}{x+1} d x=\frac{1}{2}(h)\left(f_{0}+f_{8}\right) \\
\Rightarrow T_{1}=\frac{1}{2}(1)\left(1+\frac{1}{2}\right)=0.75
\end{gathered}
$$

Using trapezoidal rule with 2 subdivisions.

$$
h=\frac{1-0}{2}=\frac{1}{2}
$$




$$
\begin{aligned}
& T_{2}=\int_{0}^{1} \frac{1}{x+1} d x=\frac{1}{2}(h)\left(f_{0}+2 f_{4}+f_{8}\right) \\
& \Rightarrow T_{2}=\frac{1}{2}\left(\frac{1}{2}\right)\left(1+2\left(\frac{2}{3}\right)+\frac{1}{2}\right)=0.708333333
\end{aligned}
$$

Using trapezoidal rule with 4 subdivisions.

$$
\begin{gathered}
h=\frac{1-0}{4}=\frac{1}{4} \\
T_{4}=\int_{0}^{1} \frac{1}{x+1} d x=\frac{1}{2}(h)\left(f_{0}+2\left(f_{2}+f_{4}+f_{6}\right)+f_{8}\right) \\
\Rightarrow T_{4}=\frac{1}{2}\left(\frac{1}{4}\right)\left(1+2\left(\frac{4}{5}+\frac{2}{3}+\frac{4}{7}\right)+\frac{1}{2}\right)=0.697023809
\end{gathered}
$$

Using trapezoidal rule with 8 subdivisions.

$$
\begin{gathered}
h=\frac{1-0}{8}=\frac{1}{8} \\
T_{8}=\int_{0}^{1} \frac{1}{x+1} d x=\frac{1}{2}(h)\left(f_{0}+2\left(\begin{array}{l}
f_{1}+f_{2}+f_{3}+f_{4}+f_{5}+ \\
f_{6}+f_{7}
\end{array}\right)+f_{8}\right) \\
\Rightarrow T_{8}=\frac{1}{2}\left(\frac{1}{8}\right)\left(1+2\left(\frac{8}{9}+\frac{4}{5}+\frac{8}{11}+\frac{2}{3}+\frac{8}{13}+\frac{4}{7}+\frac{8}{15}\right)+\frac{1}{2}\right) \\
=0.69412185
\end{gathered}
$$

Now, using Romberg approximation, we apply the iterative formula below to obtain the Romberg extrapolates as shown below

$$
T_{2 N, i+1}=\frac{4 T_{2 N, i}-T_{N, i}}{3}
$$

Table 4. Romberg Integration Iterative Formula.

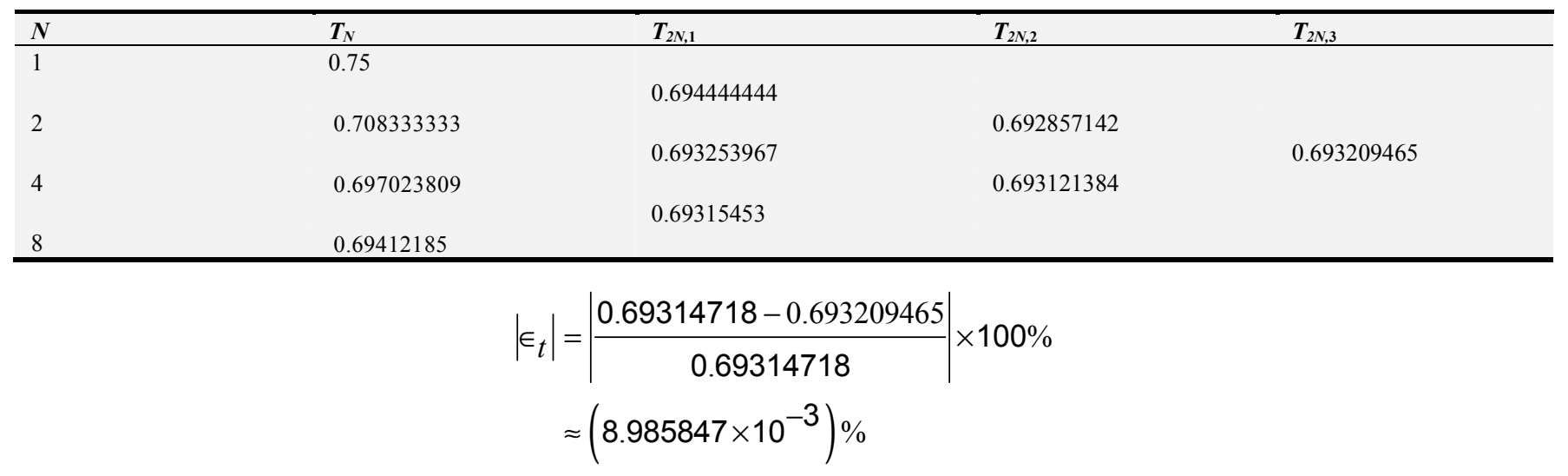

Using Boole's rule with $n=4$

$$
\begin{gathered}
h=\frac{1}{4}=0.25 \\
I=\frac{2 h}{45}\left(7 y_{0}+32 y_{1}+12 y_{2}+32 y_{3}+7 y_{4}\right)
\end{gathered}
$$




$$
\begin{gathered}
I=\frac{2 \times(0.25)}{45}(7 \times 1+32 \times 0.8+12 \times 0.66667+32 \times 0.571429+7 \times 0.5) \\
I=0.693175 \\
\left|\epsilon_{t}\right|=\left|\frac{0.69314718-0.693175}{0.69314718}\right| \times 100 \% \\
\approx\left(4.013578 \times 10^{-3}\right) \%
\end{gathered}
$$

Using Boole's rule with $n=8$

$$
\begin{gathered}
h=\frac{1}{8}=0.125 \\
I=\frac{2 h}{45}\left[7 y_{0}+32\left(y_{1}+y_{3}+y_{5}+y_{7}\right)+12\left(y_{2}+y_{6}\right)+14 y_{4}+7 y_{8}\right]
\end{gathered}
$$$$
I=\frac{2 \times(0.125)}{45}[(7 \times 1)+32(0.88889+0.727273+0.615385+0.53333)+12(0.8+0.571429)+(14 \times 0.66667)+(7 \times 0.5)]
$$$$
I=0.693148
$$$$
\left|\epsilon_{t}\right|=\left|\frac{0.69314718-0.693148}{0.69314718}\right| \times 100 \%
$$$$
\approx\left(1.04088 \times 10^{-4}\right) \%
$$

Using weddles 's rule with $n=6$

$$
\begin{gathered}
h=\frac{1}{6}=0.16667 \\
I=\frac{3 h}{10}\left[y_{0}+5 y_{1}+y_{2}+6 y_{3}+y_{4}+5 y_{5}+y_{6}\right] \\
I=\frac{3 \times 0.1667}{10}[1+5 \times 0.857143+0.75+6 \times 0.66667+0.6+5 \times 0.545454+0.5] \\
I=0.693149114 \\
\left|\epsilon_{t}\right|=\left|\frac{0.69314718-0.693149114}{0.69314718}\right| \times 100 \% \\
\approx\left(2.79072 \times 10^{-4}\right) \%
\end{gathered}
$$

Using the 2-point DIAF

$$
\mathrm{N}=2 ; h=\frac{1-0}{4(2)}=\frac{1}{8} ; f(x)=\frac{1}{x+1} ; w(x)=1
$$

So that, $I=\int_{0}^{1} \frac{1}{x+1} d x=\frac{2\left(\frac{1}{8}\right)}{45} \sum_{K=0}^{8} \lambda_{k} f\left(\frac{k}{8}\right)$

Then for the 2-point DIAF, the weights are given by $\lambda_{k}=\left\{\begin{array}{l}7, \text { if }(k=\{0,8\}) \\ y, \text { otherwise }\end{array}\right.$ 
where $y=\left[32+(-1)^{k-1}\left(\frac{\left(2^{k}+(-2)^{k}\right)}{\left\{\frac{r}{2} \frac{2^{k-1}}{5}+\left(1-\frac{r}{2}\right) \frac{2^{k}}{9}\right\}}\right)\right]$

and $r=k \bmod 4$

$$
\begin{aligned}
& \lambda_{0}=7 \\
& \lambda_{1}=\left[32+(-1)^{1-1}\left(\frac{\left(2^{1}+(-2)^{1}\right)}{\left\{\frac{1}{2} \frac{2^{1-1}}{5}+\left(1-\frac{1}{2}\right) \frac{2^{1}}{9}\right\}}\right] \lambda_{2}=\left[32+(-1)^{2-1}\left(\frac{\left(2^{2}+(-2)^{2}\right)}{\left\{\frac{2}{2} \frac{2^{2-1}}{5}+\left(1-\frac{2}{2}\right) \frac{2^{2}}{9}\right\}}\right]\right.\right. \\
& =32+\left(\frac{0}{\frac{1}{2}\left(\frac{2^{0}}{5}\right)}\right)=32 \quad=32-\left(\frac{2^{3}}{\left(\frac{2}{5}\right)}\right)=12 \\
& \lambda_{3}=\left[32+(-1)^{3-1}\left(\frac{\left(2^{3}+(-2)^{3}\right)}{\left\{\frac{3}{2} \frac{2^{3-1}}{5}+\left(1-\frac{3}{2}\right) \frac{2^{3}}{9}\right\}}\right)\right] \\
& =32+\left(\frac{0}{\frac{3}{2} \frac{2^{3-1}}{5}+\left(1-\frac{3}{2}\right) \frac{2^{3}}{9}}\right)=32 \\
& \lambda_{4}=\left[32+(-1)^{4-1}\left(\frac{\left(2^{4}+(-2)^{4}\right)}{\left\{\frac{0}{2} \frac{2^{4-1}}{5}+\left(1-\frac{0}{2}\right) \frac{2^{4}}{9}\right\}}\right)\right] \\
& =32-\left(\frac{2^{5}}{\left(\frac{2^{4}}{9}\right)}\right)=14
\end{aligned}
$$

Similarly, it follows that on substitution:

$$
\lambda_{5}=32, \lambda_{6}=12, \lambda_{7}=32, \lambda_{8}=7
$$

Thus, 


$$
\begin{aligned}
& \Rightarrow I=\frac{2\left(\frac{1}{8}\right)}{45}\left\{+32\left(\frac{1}{\frac{3}{8}+1}\right)+14\left(\frac{1}{\frac{4}{8}+1}\right)+32\left(\frac{1}{\frac{1}{8}+1}\right)+32\left(\frac{1}{\frac{1}{8}+1}\right)+12\left(\frac{1}{\frac{2}{8}+1}\right)\right\} \\
&\left.+12\left(\frac{1}{\frac{6}{8}+1}\right)+32\left(\frac{1}{\frac{7}{8}+1}\right)+7\left(\frac{1}{\frac{8}{8}+1}\right)\right\} 0.693147901 \\
&\left|\epsilon_{t}\right|=\left|\frac{0.69314718-0.693147901}{0.69314718}\right| \times 100 \% \\
& \approx\left(1.0394 \times 10^{-4}\right) \% \\
& \int_{1}^{2} x \sqrt{x+1} d x
\end{aligned}
$$

The exact solution of

$$
I=\int_{1}^{2} x \sqrt{x+1} d x
$$

analytically is as follows

Using integration by parts, put

$$
\left.\begin{array}{lc}
u=x & d v=\sqrt{x+1} d x \\
\Rightarrow d u=d x & \Rightarrow v=2 / 3(x+1)^{3 / 2}
\end{array}\right\}
$$

Then by integration by parts, it follows that

$$
\begin{aligned}
& I=2 / 3 x(x+1) 3 / 2]_{1}^{2}-\int_{1}^{2} 2 / 3(x+1) 3 / 2 d x \\
& \Rightarrow I=[2 / 3 x(x+1) 3 / 2-4 / 15(x+1) 5 / 2]_{1}^{2} \\
& =2.771281292-0.3771236166 \\
& \Rightarrow I=2.394157675
\end{aligned}
$$

Using Gauss-Chebyshev 3 point formula

$$
I=\int_{1}^{2} x \sqrt{x+1} d x
$$

We first transform the interval $[1,2]$ onto $[-1,1]$ using the transformation $x=\left(\frac{b-a}{2}\right) t+\left(\frac{b+a}{2}\right)$, where $a=1$ and $b=2 \Rightarrow x=1 / 2(t+3)$,

$$
\Rightarrow d x=1 / 2 d t
$$


Substituting equation $(i i) *$ into $(i) *$ yields, $I=\int_{-1}^{1} 1 / 4 \sqrt{2}(t+3)(t+5)^{1 / 2} d t$.

Then by Gauss-Chebyshev 3 point formula $I=\int_{-1}^{1} f(t) d t=\frac{\pi}{3}\left[f\left(\frac{\sqrt{3}}{2}\right)+f(0)+f\left(-\frac{\sqrt{3}}{2}\right)\right] \quad$ here $\frac{f(t)}{\sqrt{1-t^{2}}}=1 / 4 \sqrt{2}(t+3)(t+5)^{1 / 2}$

$\Rightarrow f(t)=1 / 4 \sqrt{2}(t+3) \sqrt{(t+5)\left(1-t^{2}\right)}$

$$
\begin{aligned}
& \Rightarrow I=\frac{\pi}{3} \cdot 1 / 4 \sqrt{2}\left[\begin{array}{l}
\left(\frac{\sqrt{3}}{2}+3\right) \sqrt{\left(\frac{\sqrt{3}}{2}+5\right)\left(1-\left(\frac{\sqrt{3}}{2}\right)^{2}\right)} \\
+\left(\left(-\frac{\sqrt{3}}{2}\right)+3\right), \sqrt{(0+5)\left(1-0^{2}\right)}
\end{array}\right] \times 100 \% \\
& =\frac{\pi}{12 \sqrt{2}}\left[\begin{array}{l}
4.681733381+6.708203932 \\
+2.16941756
\end{array}\right] \\
& =2.510109434
\end{aligned}
$$

Using Gauss-Legendre 3 point formula

$$
I=\int_{1}^{2} x \sqrt{x+1} d x
$$

We first transform the interval $[1,2]$ onto $[-1,1]$

using the transformation $x=\left(\frac{b-a}{2}\right) t+\left(\frac{b+a}{2}\right)$,

where $a=1$

and $b=2 \Rightarrow x=1 / 2(t+3)$,

$$
\Rightarrow d x=1 / 2 d t
$$

Substituting equation $(i i) *$ into $(i) *$ yields, $I=\int_{-1}^{1} 1 / 4 \sqrt{2}(t+3)(t+5)^{1 / 2} d t$.

Then by Gauss-Legendre 3 point formula $I=\int_{-1}^{1} f(t) d t=\frac{1}{9}\left[5 f\left(-\sqrt{\frac{3}{5}}\right)+8 f(0)+5 f\left(\sqrt{\frac{3}{5}}\right)\right] \quad, \quad$ here $f(t)=1 / 4 \sqrt{2}(t+3)(t+5)^{1 / 2}$.

Thus,

$$
\begin{aligned}
& \Rightarrow I=\frac{1}{9} \cdot 1 / 4 \sqrt{2}\left[\begin{array}{l}
5\left(\left(-\sqrt{\frac{3}{5}}\right)+3\right)\left(\left(-\sqrt{\frac{3}{5}}\right)+5\right)^{1 / 2}+8(0+3)(0+5) / 2 \\
+5\left(\sqrt{\frac{3}{5}}+3\right)\left(\sqrt{\frac{3}{5}}+5\right)^{1 / 2}
\end{array}\right] \\
& =\frac{1}{36 \sqrt{2}}\left[\begin{array}{l}
22.87245718+53.66563146 \\
+45.35251595
\end{array}\right] \\
& =2.394157585
\end{aligned}
$$




$$
\begin{aligned}
& \left|\varepsilon_{t}\right|=\left|\frac{2.394157675-2.394157585}{2.394157675}\right| \times 100 \% \\
& \approx 3.74926 \times 10^{-6} \%
\end{aligned}
$$

Using Simpson's 3/8 rule with 8 subdivisions

$$
h=\frac{2-1}{8}=\frac{1}{8} ; f(x)=x \sqrt{x+1}
$$

Table 5. Values of $x$ against $f(x)=x \sqrt{x+1}$.

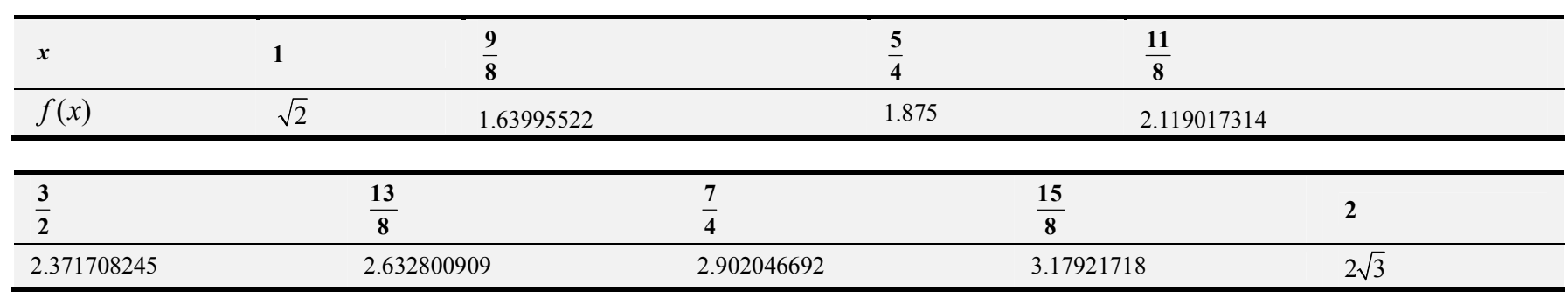

Then by Sympson's $3 / 8$ rule.

$$
\int_{a}^{b} f(x) d x=\frac{3 h}{8}\left(\begin{array}{l}
f_{0}+3\left(f_{1}+f_{2}+f_{4}+f_{5}+f_{7}+f_{8}+---+f_{3 N-2}\right. \\
\left.+f_{3 N-1}\right)+2\left(f_{3}+f_{6}+f_{9}+---+f_{3 N-3}\right)+f_{3 N}
\end{array}\right)
$$

Thus for 8 subdivisions we have,

$$
\begin{gathered}
I=\int_{1}^{2} x \sqrt{x+1} d x=\frac{3 h}{8}\left(\begin{array}{l}
f_{0}+3\left(\begin{array}{l}
f_{1}+f_{2}+f_{4} \\
+f_{5}+f_{7}
\end{array}\right) \\
+2\left(f_{3}+f_{6}\right)+f_{8}
\end{array}\right) \\
I=\int_{1}^{2} x \sqrt{x+1} d x=\frac{3}{64}\left(\begin{array}{l}
\sqrt{2}+3\left(\begin{array}{l}
1.63995522+1.875 \\
+2.371708245+2.902046692 \\
+3.17921718
\end{array}\right)+ \\
2(2.119017314+2.632800909)+2 \sqrt{3}
\end{array}\right) \\
I=2.357143764 \\
\quad\left|\varepsilon_{t}\right|=\left|\frac{2.394157675-2.357143764}{2.394157675}\right| \times 100 \% \\
\approx 1.546009746 \%
\end{gathered}
$$

\begin{tabular}{|c|c|c|c|c|c|c|}
\hline$x$ & 1 & $\frac{9}{8}$ & $\frac{5}{4}$ & $\frac{11}{8}$ & & 2 \\
\hline$f(x)$ & $\sqrt{2}$ & 1.63995522 & 1.875 & 2.119017314 & & $2 \sqrt{3}$ \\
\hline$\underline{3}$ & & 13 & 7 & & 15 & \\
\hline$\overline{2}$ & & $\overline{8}$ & $\overline{4}$ & & $\overline{8}$ & \\
\hline 2.371708245 & & 2.632800909 & 2.902046692 & & 3.17921718 & \\
\hline
\end{tabular}

Using Romberg Approximation

$$
h=\frac{2-1}{8}=\frac{1}{8} ; f(x)=x \sqrt{x+1}
$$

Table 6. Values of $x$ against $f(x)=x \sqrt{x+1}$ for Romberg Integration. 
Using trapezoidal rule with 1 subdivision.

$$
\begin{gathered}
h=\frac{2-1}{1}=1 \\
T_{1}=\int_{1}^{2} x \sqrt{x+1} d x=\frac{1}{2} h\left(f_{0}+f_{8}\right) \\
\Rightarrow T_{1}=\frac{1}{2} \cdot 1(\sqrt{2}+2 \sqrt{3}) \\
=2.439157589
\end{gathered}
$$

Using trapezoidal rule with 2 subdivisions.

$$
\begin{gathered}
h=\frac{2-1}{2}=\frac{1}{2} \\
T_{2}=\int_{1}^{2} x \sqrt{x+1} d x=\frac{1}{2} h\left(f_{0}+2 f_{4}+f_{8}\right) \\
\Rightarrow T_{2}=\frac{1}{2}(1 / 2)(\sqrt{2}+2(2.371708245)+2 \sqrt{3}) \\
=2.405432917
\end{gathered}
$$

Using trapezoidal rule with 4 subdivisions.

$$
\begin{gathered}
h=\frac{2-1}{4}=\frac{1}{4} \\
T_{4}=\int_{1}^{2} x \sqrt{x+1} d x=\frac{1}{2} h\left(f_{0}+2\left(f_{2}+f_{4}+f_{6}\right)+f_{8}\right) \\
\Rightarrow T_{4}=\frac{1}{2}(1 / 4)\left(\sqrt{2}+2\left(\begin{array}{l}
1.875+2.371708245 \\
+2.902046692
\end{array}\right)+2 . \sqrt{3}\right) \\
=2.396978131
\end{gathered}
$$

Using trapezoidal rule with 8 subdivisions.

$$
\left.\begin{array}{c}
h=\frac{2-1}{8}=\frac{1}{8} \\
T_{8}=\int_{1}^{2} x \sqrt{x+1} d x=\frac{1}{2} h\left(\begin{array}{l}
f_{1}+f_{2}+f_{3} \\
+f_{4}+f_{5}+f_{6} \\
+f_{7}
\end{array}\right)+f_{8}
\end{array}\right)
$$

Now, using Romberg approximation, we apply the iterative formula below to obtain the Romberg extrapolates as shown below

$$
T_{2 N, i+1}=\frac{4 T_{2 N, i}-T_{N, i}}{3}
$$


Table 7. Values of $x$ against $f(x)=x \sqrt{x+1}$ for Romberg Iterative Integration.

\begin{tabular}{lllll}
\hline $\boldsymbol{N}$ & $\boldsymbol{T}_{\boldsymbol{N}}$ & $\boldsymbol{T}_{2 N, 1}$ & $\boldsymbol{T}_{2 N, 2}$ & $\boldsymbol{T}_{2 N, 3}$ \\
\hline 1 & 2.439157589 & 2.39419136 & & \\
2 & 2.405432917 & 2.394159869 & 2.394149372 & 2.394159716 \\
4 & 2.396978131 & 2.394157815 & 2.39415713 & \\
\hline & 2.394862894 & & \\
\hline
\end{tabular}

So the Romberg approximation gives $I=\int_{1}^{2} x \sqrt{x+1} d x=2.394159716$

$$
\begin{aligned}
& \left|\mathcal{E}_{t}\right|=\left|\frac{2.394157675-2.394159716}{2.394157675}\right| \times 100 \% \\
& \approx 8.5249 \times 10^{-4} \%
\end{aligned}
$$

Using Boole's rule with $n=4$

$$
\begin{gathered}
h=\frac{1}{4}=0.25 \\
I=\frac{2 h}{45}\left(7 y_{0}+32 y_{1}+12 y_{2}+32 y_{3}+7 y_{4}\right) \\
I=\frac{2 \times(0.25)}{45}(7 \times 1.414214+32 \times 1.875+12 \times 2.371708+32 \times 2.902047+7 \times 3.464102) \\
I=2.394158 \\
\left|\varepsilon_{\mathrm{t}}\right|=\left|\frac{2.394157675-2.39415777}{2.394157675}\right| \times 100 \% \\
\approx 3.97451 \times 10^{-6} \mathrm{o} / \mathrm{o}
\end{gathered}
$$

Using Boole's rule with $n=8$

$$
\begin{gathered}
h=\frac{1}{8}=0.125 \\
I=\frac{2 h}{45}\left[7 y_{0}+32\left(y_{1}+y_{3}+y_{5}+y_{7}\right)+12\left(y_{2}+y_{6}\right)+14 y_{4}+7 y_{8}\right] \\
I=\frac{2 \times(0.125)}{45}\left(\begin{array}{l}
7 \times 1.414214)+32(1.639955+2.119017+2.632801+3.179217) \\
+12(1.875+2.902047)+(14 \times 2.371708)+(7 \times 3.464102)
\end{array}\right) \\
\quad I=2.394158 \\
\quad \varepsilon_{\mathrm{t}}|=| \frac{2.394157675-2.394158}{2.394157675} \mid \times 100 \% \\
\quad 0.97451 \times 10^{-6} \mathrm{o} / \mathrm{o}
\end{gathered}
$$

Using weddles 's rule with $n=6$ 


$$
\begin{gathered}
h=\frac{1}{6}=0.16667 \\
I=\frac{3 h}{10}\left[y_{0}+5 y_{1}+y_{2}+6 y_{3}+y_{4}+5 y_{5}+y_{6}\right] \\
I=\frac{3 \times 0.1667}{10}\left[\begin{array}{l}
1.414214+5 \times 1.717349+2.036766+6 \times 2.371778 \\
+2.721729+5 \times 3.086037+3.464182
\end{array}\right] I=2.394703395 \\
\left|\varepsilon_{\mathrm{t}}\right|=\left|\frac{2.394157675-2.394703395}{2.394157675}\right| \times 100 \% \\
\approx 2.2793832 \times 10^{-2} \%
\end{gathered}
$$

Using the 2-point DIAF

$\mathrm{N}=2 ; h=\frac{2-1}{4(2)}=\frac{1}{8} ; f(x)=x \sqrt{x+1} ; w(x)=1$

So that, $I=\int_{1}^{2} x \sqrt{x+1} d x=\frac{2(1 / 8)}{45} \sum_{k=0}^{8} \lambda_{k} f\left(1+\frac{k}{8}\right)$

Then for the 2-point DIAF, the weights are given by $\lambda_{k}=\left\{\begin{array}{l}7, \text { if }(k=\{0,8\}) \\ y, \text { otherwise }\end{array}\right.$ where

$$
y=\left[32+(-1)^{k-1}\left(\frac{\left(2^{k}+(-2)^{k}\right)}{\left\{\frac{r}{2} \frac{2^{k-1}}{5}+\left(1-\frac{r}{2}\right) \frac{2^{k}}{9}\right\}}\right)\right]
$$

and $r=k \bmod 4$

$$
\begin{aligned}
\lambda_{1}=\left[32+(-1)^{1-1}\left(\frac{\left.2^{1}+(-2)^{1}\right)}{\left\{\frac{1}{2} \frac{2^{1-1}}{5}+\left(1-\frac{1}{2}\right) \frac{2^{1}}{9}\right\}}\right)\right] \lambda_{2}=\left[32+(-1)^{2-1}\left(\frac{\left(2^{2}+(-2)^{2}\right)}{\left.\left.\left\{\frac{2}{2} \frac{2^{2-1}}{5}+\left(1-\frac{2}{2}\right) \frac{2^{2}}{9}\right\}\right)\right]}\right.\right. \\
=32+\left(\frac{0}{\frac{1}{2}\left(\frac{2^{0}}{5}\right)}\right)=32 \quad=32-\left(\frac{2^{3}}{\left(\frac{2}{5}\right)}\right)=12
\end{aligned}
$$




$$
\begin{aligned}
\lambda_{3} & =\left[32+(-1)^{3-1}\left(\frac{\left(2^{3}+(-2)^{3}\right)}{\left\{\frac{3}{2} \frac{2^{3-1}}{5}+\left(1-\frac{3}{2}\right) \frac{2^{3}}{9}\right\}}\right)\right] \\
& =32+\left(\frac{0}{\frac{3}{2} \frac{2^{3-1}}{5}+\left(1-\frac{3}{2}\right) \frac{2^{3}}{9}}\right)=32 \\
\lambda_{4} & =\left[32+(-1)^{4-1}\left(\frac{\left(2^{4}+(-2)^{4}\right)}{\left\{\frac{0}{2} \frac{2^{4-1}}{5}+\left(1-\frac{0}{2}\right) \frac{2^{4}}{9}\right\}}\right)\right] \\
& =32-\left(\frac{2^{5}}{\left(\frac{2^{4}}{9}\right)}\right)=14
\end{aligned}
$$

Similarly, it follows that on substitution:

$$
\lambda_{5}=32, \lambda_{6}=12, \lambda_{7}=32, \lambda_{8}=7
$$

Thus,

$$
\begin{aligned}
& \Rightarrow I=\frac{2(1 / 8)}{45}\left\{\begin{array}{l}
7(1 \sqrt{1+1})+32(9 / 8 \sqrt{9 / 8+1}) \\
+12(5 / 4 \sqrt{5 / 4+1})+32(11 / 8 \sqrt{11 / 8+1}) \\
+14(3 / 2 \sqrt{3 / 2+1})+32(13 / 8 \sqrt{13 / 8+1}) \\
+12(7 / 4 \sqrt{7 / 4+1})+32(15 / 8 \sqrt{15 / 8+1}) \\
+7(2 \sqrt{2+1})
\end{array}\right\} \\
& \Rightarrow I=\frac{1}{180}\left(\begin{array}{l}
7(\sqrt{2})+32(1.63995522)+12(1.875) \\
+32(2.119017314)+14(2.371708245) \\
+32(2.632800909)+12(2.902046692) \\
+32(3.17921718)+7(2 \sqrt{3})
\end{array}\right) \\
& \left.=2.394157677 \quad \begin{array}{c}
2.394157675 \\
\int_{0}^{1} x e^{x^{2}} d x
\end{array}|=| \frac{2.394157675-2.394157677}{2}\right) \times 100 \% \\
& \approx 8.3537 \times 10^{-8} \%
\end{aligned}
$$

The exact solution of

$$
I=\int_{0}^{1} x e^{x^{2}} d x-------(i)
$$


analytically is as follows

$$
\text { Put } \left.\begin{array}{l}
u=x^{2} \\
\Rightarrow d u=2 x d x
\end{array}\right\}---------(i i)
$$

Then substituting (ii) into (i) yields

$$
\begin{aligned}
& I=\int_{0}^{1} 1 / 2 e^{u} d u \\
& \Rightarrow I=1 / 2\left[e^{u}\right]_{0}^{1}=1 / 2(e-1) \\
& \Rightarrow I=0.8591409142
\end{aligned}
$$

Using Gauss-Chebyshev 3 point formula

$$
I=\int_{0}^{1} x e^{x^{2}} d x
$$

We first transform the interval $[0,1]$ onto $[-1,1]$ using the transformation $x=\left(\frac{b-a}{2}\right) t+\left(\frac{b+a}{2}\right)$, where $a=0$ and $b=1 \Rightarrow x=1 / 2 t+1 / 2$,

$$
\Rightarrow d x=1 / 2 d t
$$

Substituting equation $(i i) *$ into $(i) *$ yields, $I=\int_{-1}^{1} 1 / 4(t+1) \cdot e^{1 / 4(t+1)^{2}} d t$.

Then by Gauss-Chebyshev 3 point formula $I=\int_{-1}^{1} f(t) d t=\frac{\pi}{3}\left[f\left(\frac{\sqrt{3}}{2}\right)+f(0)+f\left(-\frac{\sqrt{3}}{2}\right)\right]$,

here $\frac{f(t)}{\sqrt{1-t^{2}}}=1 / 4(t+1) \cdot e^{1 / 4^{(t+1)^{2}}}$

$$
\begin{aligned}
& \Rightarrow f(t)=1 / 4(t+1) \cdot e^{1 / 4(t+1)^{2}} \sqrt{\left(1-t^{2}\right)} \\
& \left(\frac{\sqrt{3}}{2}+1\right) \cdot e^{1 / 4\left(\frac{\sqrt{3}}{2}+1\right)^{2}} \sqrt{\left(1-\left(\frac{\sqrt{3}}{2}\right)^{2}\right)} \\
& \Rightarrow I=\frac{\pi}{3} \cdot 1 / 4+(0+1) \cdot e^{1 / 4^{(0+1)^{2}}} \sqrt{\left(1-0^{2}\right)}
\end{aligned}
$$

Thus,

$$
\left[+\left(-\frac{\sqrt{3}}{2}+1\right) e^{1 / 4\left(-\frac{\sqrt{3}}{2}+1\right)^{2}} \sqrt{\left(1-\left(-\frac{\sqrt{3}}{2}\right)^{2}\right)}\right]
$$

$$
\begin{aligned}
& =\frac{\pi}{12}(2.228160234+1.284025417+0.06728856552) \\
& =0.9371041584
\end{aligned}
$$

$$
\begin{aligned}
& \left|\varepsilon_{t}\right|=\left|\frac{0.8591409142-0.9371041584}{0.8591409142}\right| \times 100 \% \\
& \approx 9.07455842 \%
\end{aligned}
$$

Using Gauss-Legendre 3 point formula 


$$
I=\int_{0}^{1} x e^{x^{2}} d x
$$

We first transform the interval $[0,1]$ onto $[-1,1]$

using the transformation $x=\left(\frac{b-a}{2}\right) t+\left(\frac{b+a}{2}\right)$, where $a=0$

and $b=1 \Rightarrow x=1 / 2 t+1 / 2$,

$$
\Rightarrow d x=1 / 2 d t
$$

Substituting equation $(i i) *$ into $(i) *$ yields, $I=\int_{-1}^{1} 1 / 4(t+1) \cdot e^{1 / 4(t+1)^{2}} d t$.

Then by Gauss-Legendre 3 point formula $I=\int_{-1}^{1} f(t) d t=\frac{1}{9}\left[5 f\left(-\sqrt{\frac{3}{5}}\right)+8 f(0)+5 f\left(\sqrt{\frac{3}{5}}\right)\right]$, here $f(t)=1 / 4(t+1) \cdot e^{1 /(t+1)^{2}}$.

Thus,

$$
\begin{aligned}
& \Rightarrow I=\frac{1}{9} \cdot 1 / 4\left[\begin{array}{l}
5\left(-\sqrt{\frac{3}{5}}+1\right) \cdot e^{1 / 4\left(-\sqrt{\frac{3}{5}}+1\right)^{2}} \\
+8(0+1) \cdot e^{1 / 4(0+1)^{2}} \\
+5\left(\sqrt{\frac{3}{5}}+1\right) \cdot e^{1 / 4\left(\sqrt{\frac{3}{5}}+1\right)^{2}}
\end{array}\right] \\
& =\frac{1}{36}\left[\begin{array}{l}
1.14142294+10.27220333 \\
+19.49795163
\end{array}\right] \\
& =0.8586549417 \\
& \left|\varepsilon_{t}\right|=\left|\frac{0.8591409142-0.8586549417}{0.8591409142}\right| \times 100 \% \\
& \approx 5.6564938 \times 10^{-1} \%
\end{aligned}
$$

\begin{tabular}{|c|c|c|c|c|}
\hline$x$ & $\mathbf{0}$ & $\frac{1}{8}$ & $\frac{1}{4}$ & $\frac{3}{8}$ \\
\hline$f(x)$ & 0 & 0.1269684636 & 0.2661236147 & 0.4316223543 \\
\hline$\frac{1}{2}$ & & & $\frac{5}{8}$ & 1 \\
\hline 0.6420127083 & & & 0.9236901221 & 2.718281828 \\
\hline
\end{tabular}

Using Simpson's $3 / 8$ rule with 8 subdivisions

$$
h=\frac{1-0}{8}=\frac{1}{8} ; f(x)=x e^{x^{2}}
$$

Table 8. Values of $x$ against $f(x)=x e^{x^{2}}$ for Simpson's $3 / 8$ rule with 8 subdivisions.

Then by Sympson's 3/8 rule.

$$
\int_{a}^{b} f(x) d x=\frac{3 h}{8}\left(\begin{array}{l}
f_{0}+3\left(f_{1}+f_{2}+f_{4}+f_{5}+f_{7}+f_{8}+---+f_{3 N-2}\right. \\
\left.+f_{3 N-1}\right)+2\left(f_{3}+f_{6}+f_{9}+---+f_{3 N-3}\right)+f_{3 N}
\end{array}\right)
$$


Thus for 8 subdivisions we have,

$$
\begin{aligned}
& I=\int_{0}^{1} x e^{x^{2}} d x=\frac{3 h}{8}\left(\begin{array}{l}
f_{0}+3\left(\begin{array}{l}
f_{1}+f_{2}+f_{4} \\
+f_{5}+f_{7} \\
+2\left(f_{3}+f_{6}\right)+f_{8}
\end{array}\right)
\end{array}\right) \\
& I=\int_{0}^{1} x e^{x^{2}} d x=\frac{3}{64}\left(\begin{array}{l}
0+3\left(\begin{array}{l}
0.1269684636+0.2661236147 \\
+0.6420127083+ \\
0.9236901221+1.881545676
\end{array}\right) \\
+2(0.4316223543+1.316290993)+2.718281828
\end{array}\right) \\
& I=0.8313342317 \\
& \left|\varepsilon_{t}\right|=\left|\frac{0.8591409142-0.8313342317}{0.8591409142}\right| \times 100 \% \\
& \approx 3.236568305 \%
\end{aligned}
$$

Using Romberg Approximation

$$
h=\frac{1-0}{8}=\frac{1}{8} ; f(x)=x e^{x^{2}}
$$

Table 9. Values of $x$ against $f(x)=x e^{x^{2}}$ for Romberg Approximation with 8 subdivisions

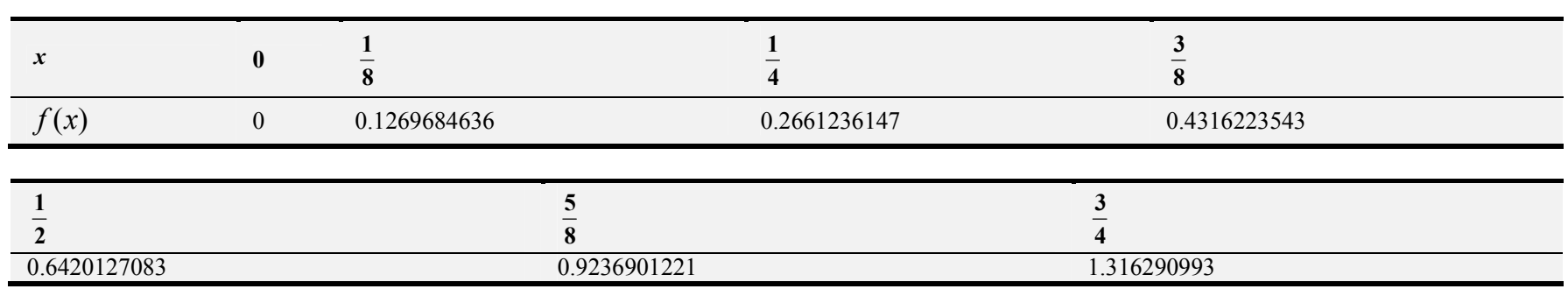

Using trapezoidal rule with 1 subdivision.

$$
\begin{gathered}
h=\frac{1-0}{1}=1 \\
T_{1}=\int_{0}^{1} x e^{x^{2}} d x=\frac{1}{2} h\left(f_{0}+f_{8}\right) \\
\Rightarrow T_{1}=\frac{1}{2} \cdot 1(0+e) \\
=1.359140914
\end{gathered}
$$

Using trapezoidal rule with 2 subdivisions.

$$
\begin{gathered}
h=\frac{1-0}{2}=\frac{1}{2} \\
T_{2}=\int_{0}^{1} x e^{x^{2}} d x=\frac{1}{2} h\left(f_{0}+2 f_{4}+f_{8}\right) \\
\Rightarrow T_{2}=\frac{1}{2}(1 / 2)(0+2(0.6420127083)+2.718281828) \\
=1.000576811
\end{gathered}
$$


Using trapezoidal rule with 4 subdivisions.

$$
\begin{gathered}
h=\frac{1-0}{4}=\frac{1}{4} \\
T_{4}=\int_{0}^{1} x e^{x^{2}} d x=\frac{1}{2} h\left(f_{0}+2\left(f_{2}+f_{4}+f_{6}\right)+f_{8}\right) \\
\Rightarrow T_{4}=\frac{1}{2}(1 / 4)\left(0+2\left(\begin{array}{l}
0.266123647 \\
+0.6420127083+1.31629098
\end{array}\right)+e\right) \\
=0.8958920576
\end{gathered}
$$

Using trapezoidal rule with 8 subdivisions.

$$
\begin{aligned}
& h=\frac{1-0}{8}=\frac{1}{8} \\
& T_{8}=\int_{0}^{1} x e^{x^{2}} d x=\frac{1}{2} h\left(f_{0}+2\left(\begin{array}{l}
f_{1}+f_{2}+f_{3} \\
+f_{4}+f_{5}+f_{6} \\
+f_{7}
\end{array}\right)+f_{8}\right) \\
& \Rightarrow T 8=\frac{1}{2}(1 / 8)\left(\begin{array}{l}
\left.0+2\left(\begin{array}{lll}
0.12696846 & 36+0.26612361 & 47 \\
+0.43162235 & 43+0.6420127083 \\
+0.92369012 & 21+1.31629099 & 3 \\
+1.88154567 & 6
\end{array}\right)\right) \\
+e
\end{array}\right. \\
& =0.8684243558
\end{aligned}
$$

Now, using Romberg approximation, we apply the iterative formula below to obtain the Romberg extrapolates as shown below

$$
T_{2 N, i+1}=\frac{4 T_{2 N, i}-T_{N, i}}{3}
$$

Table 10. Values of $x$ against $f(x)=x e^{x^{2}}$ for Romberg Approximation Iterative formula with 8 subdivisions.

\begin{tabular}{lllll}
\hline $\boldsymbol{N}$ & $\boldsymbol{T}_{N}$ & $\boldsymbol{T}_{2 N, 1}$ & $\boldsymbol{T}_{2 N, 2}$ & $\boldsymbol{T}_{2 N, 3}$ \\
\hline 1 & 1.359140914 & 0.8810554433 & & \\
2 & 1.000576811 & 0.8609971398 & 0.8543110386 & 0.8601526231 \\
4 & 0.8958920576 & 0.8592684552 & 0.858692227 & \\
8 & 0.8684243558 & & & \\
\hline
\end{tabular}

So the Romberg approximation gives

$$
\begin{gathered}
I=\int_{0}^{1} x e^{x^{2}} d x=0.8601526231 \\
\left|\varepsilon_{t}\right|=\left|\frac{0.8591409142-0.8601526231}{0.8591409142}\right| \times 100 \% \\
\approx 1.17758199 \times 10^{-1} \mathrm{o} / \mathrm{o}
\end{gathered}
$$


Using Boole's rule with $n=4$

$$
\begin{gathered}
h=\frac{1}{4}=0.25 \\
I=\frac{2 h}{45}\left(7 y_{0}+32 y_{1}+12 y_{2}+32 y_{3}+7 y_{4}\right) \\
I=\frac{2 \times(0.25)}{45}(7 \times 0+32 \times 0.266124+12 \times 0.642013+32 \times 1.316291+7 \times 2.718282) \\
I=0.859659919 \\
\left|\varepsilon_{\mathrm{t}}\right|=\left|\frac{0.8591409142-0.859659919}{0.8591409142}\right| \times 100 \% \\
\approx 6.041 \times 10^{-2} \mathrm{o} / \mathrm{o}
\end{gathered}
$$

Using Boole's rule with $n=8$

$$
\begin{gathered}
h=\frac{1}{8}=0.125 \\
I=\frac{2 h}{45}\left[7 y_{0}+32\left(y_{1}+y_{3}+y_{5}+y_{7}\right)+12\left(y_{2}+y_{6}\right)+14 y_{4}+7 y_{8}\right] \\
I=\frac{2 \times(0.125)}{45}\left(\begin{array}{l}
7 \times 0+32(0.126968+0.431622+0.92369+1.881246) \\
+12(0.266124+1.316291)+(14 \times 0.642013)+(7 \times 2.718282)
\end{array}\right) \\
\quad \varepsilon_{\mathrm{t}}|=| \frac{0.8591409142-0.859153}{0.8591409142} \mid \times 100 \% \\
\approx 1.431129 \times 10^{-3} \%
\end{gathered}
$$

Using weddles 's rule with $n=6$

$$
\begin{gathered}
h=\frac{1}{6}=0.16667 \\
I=\frac{3 h}{10}\left[y_{0}+5 y_{1}+y_{2}+6 y_{3}+y_{4}+5 y_{5}+y_{6}\right]
\end{gathered}
$$$$
I=\frac{3 \times 0.1667}{10}[0+5 \times 0.171397+0.372597+6 \times 0.642205+1.040142+5 \times 1.669628+2.719913]
$$$$
I=0.859568
$$$$
\begin{aligned}
& \left|\varepsilon_{\mathrm{t}}\right|=\left|\frac{0.8591409142-0.859568}{0.8591409142}\right| \times 100 \% \\
& \approx 4.968 \times 10^{-2} \%
\end{aligned}
$$

Using the 2-point DIAF 


$$
\mathrm{N}=2 ; h=\frac{1-0}{4(2)}=\frac{1}{8} ; f(x)=x e^{x^{2}} ; w(x)=1
$$

So that, $I=\int_{0}^{1} x e^{x^{2}} d x=\frac{2(1 / 8)}{45} \sum_{k=0}^{8} \lambda_{k} f\left(\frac{k}{8}\right)$

Then for the 2-point DIAF, the weights are given by $\lambda_{k}=\left\{\begin{array}{l}7, \text { if }(k=\{0,8\}) \\ y, \text { otherwise }\end{array}\right.$ where

$$
y=\left[32+(-1)^{k-1}\left(\frac{\left(2^{k}+(-2)^{k}\right)}{\left\{\frac{r}{2} \frac{2^{k-1}}{5}+\left(1-\frac{r}{2}\right) \frac{2^{k}}{9}\right\}}\right]\right]
$$

and $r=k \bmod 4$

$$
\begin{aligned}
& \lambda_{0}=7 \\
& \lambda_{1}=\left[32+(-1)^{1-1}\left(\frac{\left(2^{1}+(-2)^{1}\right)}{\left\{\frac{1}{2} \frac{2^{1-1}}{5}+\left(1-\frac{1}{2}\right) \frac{2^{1}}{9}\right\}}\right)\right] \lambda_{2}=\left[32+(-1)^{2-1}\left(\frac{\left(2^{2}+(-2)^{2}\right)}{\left\{\frac{2}{2} \frac{2^{2-1}}{5}+\left(1-\frac{2}{2}\right) \frac{2^{2}}{9}\right\}}\right)\right] \\
& =32+\left(\frac{0}{\frac{1}{2}\left(\frac{2^{0}}{5}\right)}\right)=32 \quad=32-\left(\frac{2^{3}}{\left(\frac{2}{5}\right)}\right)=12 \\
& \lambda_{3}=\left[32+(-1)^{3-1}\left(\frac{\left(2^{3}+(-2)^{3}\right)}{\left\{\frac{3}{2} \frac{2^{3-1}}{5}+\left(1-\frac{3}{2}\right) \frac{2^{3}}{9}\right\}}\right)\right] \\
& =32+\left(\frac{0}{\frac{3}{2} \frac{2^{3-1}}{5}+\left(1-\frac{3}{2}\right) \frac{2^{3}}{9}}\right)=32 \\
& \lambda_{4}=\left[32+(-1)^{4-1}\left(\frac{\left(2^{4}+(-2)^{4}\right)}{\left\{\frac{0}{2} \frac{2^{4-1}}{5}+\left(1-\frac{0}{2}\right) \frac{2^{4}}{9}\right\}}\right)\right] \\
& =32-\left(\frac{2^{5}}{\left(\frac{2^{4}}{9}\right)}\right)=14
\end{aligned}
$$


Similarly, it follows that on substitution:

$$
\lambda_{5}=32, \lambda_{6}=12, \lambda_{7}=32, \lambda_{8}=7
$$

Thus

$$
\begin{aligned}
& \Rightarrow I=\frac{2(1 / 8)}{45}\left\{\begin{array}{l}
7\left(0 e^{0^{2}}\right)+32\left(1 / 8 e^{(1 / 8)^{2}}\right) \\
+12\left(1 / 4 e^{(1 / 4)^{2}}\right)+32\left(3 / 8 e^{(3 / 8)^{2}}\right) \\
+14\left(1 / 2 e^{(1 / 2)^{2}}\right)+32\left(5 / 8 e^{\left.(5 / 8)^{2}\right)}\right) \\
+12\left(3 / 4 e^{(3 / 4)^{2}}\right)+32\left(7 / 8 e^{(7 / 8)^{2}}\right)+7\left(e^{1}\right)
\end{array}\right\} \\
& \Rightarrow I=\frac{1}{180}\left(\begin{array}{l}
7(0)+32(0.1269684636)+ \\
12(0.2661236147)+32(0.4316223543) \\
+14(0.6420127083)+32(0.9236901221) \\
+12(1.316290993)+32(1.881545676)+7(e)
\end{array}\right) \\
& =0.8591532096 \\
& \left|\varepsilon_{t}\right|=\left|\frac{0.8591409142-0.8591532096}{0.8591409142}\right| \times 100 \% \\
& \approx 1.431123 \times 10^{-2} \% \\
& \int_{0}^{\pi / 2} e^{x} \cos x d x
\end{aligned}
$$

The exact solution of

$$
I=\int_{0}^{\pi / 2} e^{x} \cos x d x
$$

analytically is as follows

Using integration by parts, put

$$
\left.\begin{array}{ll}
u=\cos x & d v=e^{x} d x \\
\Rightarrow d u=-\sin x d x & \Rightarrow v=e^{x}
\end{array}\right\}
$$

Then by integration by parts, it follows that

$$
\left.I=e^{x} \cos x\right]_{0}^{\pi / 2}+\int_{0}^{\pi / 2} e^{x} \sin x d x
$$

Further put $\left.\begin{array}{ll}u=\sin x & d v=e^{x} d x \\ \Rightarrow d u=\cos x d x & \Rightarrow v=e^{x}\end{array}\right\}$ 


$$
\begin{aligned}
& \Rightarrow I=\left[e^{x} \cos x+e^{x} \sin x\right]_{0}^{\pi / 2}-\int_{0}^{\pi / 2} e^{x} \cos x d x \\
& \Rightarrow I=1 / 2\left[e^{x} \cos x+e^{x} \sin x\right]_{0}^{\pi / 2}=1 / 2\left(e^{\pi / 2}-1\right) \\
& \Rightarrow I=1.90523869
\end{aligned}
$$

Using Gauss-Chebyshev 3 point formula

$$
I=\int_{0}^{\pi / 2} e^{x} \cos x d x
$$

We first transform the interval $[0, \pi / 2]$ onto $[-1,1]$ using the transformation $x=\left(\frac{b-a}{2}\right) t+\left(\frac{b+a}{2}\right)$, where $a=0$ and $b=\pi / 2 \Rightarrow x=\pi / 4(t+1)$,

$$
\Rightarrow d x=\pi / 4 d t-------------(i i) *
$$

Substituting equation $(i i) *$ into $(i) *$ yields, $I=\int_{-1}^{1} \pi / 4 \cdot e^{\pi / 4^{(t+1)}} \cos (\pi / 4(t+1)) d t$.

Then by Gauss-Chebyshev 3 point formula $I=\int_{-1}^{1} f(t) d t=\frac{\pi}{3}\left[f\left(\frac{\sqrt{3}}{2}\right)+f(0)+f\left(-\frac{\sqrt{3}}{2}\right)\right]$,

here $\frac{f(t)}{\sqrt{1-t^{2}}}=\pi / 4 \cdot e^{\pi / 4(t+1)} \cos (\pi / 4(t+1))$

$$
\begin{aligned}
& \Rightarrow f(t)=\pi / 4 \cdot e^{\pi / 4(t+1)} \sqrt{1-t^{2}} \cos (\pi / 4(t+1)) \\
& \left.e^{\pi / 4\left(-\frac{\sqrt{3}}{2}+1\right)} \sqrt{1-\left(-\frac{\sqrt{3}}{2}\right)^{2}} \cos \left(\pi / 4\left(-\frac{\sqrt{3}}{2}+1\right)\right)\right] \\
& \Rightarrow I=\frac{\pi}{3} \cdot \pi / 4\left[\begin{array}{l}
+e^{\pi / 4(0+1)} \sqrt{1-0^{2}} \cos (\pi / 4(0+1)) \\
+e^{\pi / 4\left(\frac{\sqrt{3}}{2}+1\right)} \sqrt{1-\left(\frac{\sqrt{3}}{2}\right)^{2}} \cos \left(\pi / 4\left(\frac{\sqrt{3}}{2}+1\right)\right)
\end{array}\right]
\end{aligned}
$$

Thus, $=\frac{\pi^{2}}{12}\left[\begin{array}{l}0.5524070987+1.550883197 \\ +0.22738974\end{array}\right]$

$=1.916907495$

$$
\begin{aligned}
& \left|\varepsilon_{t}\right|=\left|\frac{1.90523869-1.916907495}{1.90523869}\right| \times 100 \% \\
& \approx 6.12458943 \times 10^{-1} \%
\end{aligned}
$$

Using Gauss-Legendre 3 point formula

$$
I=\int_{0}^{\pi / 2} e^{x} \cos x d x
$$

We first transform the interval $[0, \pi / 2]$ onto $[-1,1]$ using the transformation $x=\left(\frac{b-a}{2}\right) t+\left(\frac{b+a}{2}\right)$, where $a=0$ 
and $b=\pi / 2 \Rightarrow x=\pi / 4(t+1)$,

$$
\Rightarrow d x=\pi / 4 d t-------------(i i) *
$$

Substituting equation $(i i) *$ into $(i) *$ yields, $I=\int_{-1}^{1} \pi / 4 \cdot e^{\pi / 4(t+1)} \cos (\pi / 4(t+1)) d t$.

Then by Gauss-Legendre 3 point formula $I=\int_{-1}^{1} f(t) d t=\frac{1}{9}\left[5 f\left(-\sqrt{\frac{3}{5}}\right)+8 f(0)+5 f\left(\sqrt{\frac{3}{5}}\right)\right]$, here $f(t)=\pi / 4 \cdot e^{\pi / 4(t+1)} \cos (\pi / 4(t+1))$.

Thus,

$$
\begin{aligned}
& \Rightarrow I=\frac{1}{9} \cdot \pi / 4\left[\begin{array}{l}
5 . e^{\pi / 4\left(-\sqrt{\frac{3}{5}}+1\right)} \cos \left(\pi / 4\left(-\sqrt{\frac{3}{5}}+1\right)\right) \\
+8 e^{\pi / 4(0+1)} \cos (\pi / 4(0+1)) \\
\pi / 4\left(\sqrt{\frac{3}{5}}+1\right) \\
+5 e^{-1}\left(\pi / 4\left(\sqrt{\frac{3}{5}}+1\right)\right)
\end{array}\right] \\
& =\frac{\pi}{36}\left[\begin{array}{l}
5.875062405+12.40706558 \\
+3.548573471
\end{array}\right] \\
& =1.905088092
\end{aligned}
$$

$$
\begin{aligned}
& \left|\varepsilon_{t}\right|=\left|\frac{1.90523869-1.905088092}{1.90523869}\right| \times 100 \% \\
& \approx 7.90443 \times 10^{-3} \%
\end{aligned}
$$

\begin{tabular}{|c|c|c|c|c|c|}
\hline$x$ & 0 & $\frac{\pi}{16}$ & $\frac{\pi}{8}$ & $\frac{3 \pi}{16}$ & $\frac{\pi}{4}$ \\
\hline$f(x)$ & 1 & 1.19356881 & 1.368240338 & 1.498535197 & 1.550883197 \\
\hline $5 \pi$ & & & & $7 \pi$ & $\pi /$ \\
\hline 16 & & & & $\overline{16}$ & \\
\hline 1.482881951 & & & & 0.7711704511 & 0 \\
\hline
\end{tabular}

Using Simpson's $3 / 8$ rule with 8 subdivisions

$$
h=\frac{\pi / 2-0}{8}=\frac{\pi}{16} ; f(x)=e^{x} \cos x
$$

Table 11. Values of $x$ against $f(x)=e^{x} \cos x$ for Simpson's $3 / 8$ rule with 8 subdivisions.

Then by Sympson's 3/8 rule.

$$
\int_{a}^{b} f(x) d x=\frac{3 h}{8}\left(\begin{array}{l}
f_{0}+3\left(f_{1}+f_{2}+f_{4}+f_{5}+f_{7}+f_{8}+---+f_{3 N-2}\right. \\
\left.+f_{3 N-1}\right)+2\left(f_{3}+f_{6}+f_{9}+---+f_{3 N-3}\right)+f_{3 N}
\end{array}\right)
$$


Thus for 8 subdivisions we have,

$$
\begin{aligned}
& I=\int_{0}^{\pi / 2} e^{x} \cos x d x=\frac{3 h}{8}\left(\begin{array}{l}
f_{0}+3\left(\begin{array}{l}
f_{1}+f_{2} \\
+f_{4}+f_{5}+f_{7}
\end{array}\right) \\
+2\left(f_{3}+f_{6}\right)+f_{8}
\end{array}\right) \\
& I=\int_{0}^{\pi / 2} e^{x} \cos x d x=\frac{3 \pi}{128}\left(\begin{array}{l}
1.19356881+1.368240338 \\
1+3\left(\begin{array}{l}
1.550883197+1.482881951 \\
+0.7711704511
\end{array}\right) \\
+2(1.498535197+1.243027662)+0
\end{array}\right) \\
& I=1.883730368 \\
& \left|\varepsilon_{t}\right|=\left|\frac{1.90523869-1.883730368}{1.90523869}\right| \times 100 \% \\
& \approx 1.128904325 \%
\end{aligned}
$$

\begin{tabular}{|c|c|c|c|c|c|}
\hline$x$ & $\mathbf{0}$ & $\frac{\pi}{16}$ & $\frac{\pi}{8}$ & $\frac{3 \pi}{16}$ & \\
\hline$f(x)$ & 1 & 1.19356881 & 1.368240338 & 1.498535197 & \\
\hline$\frac{\pi}{4}$ & & $\frac{5 \pi}{16}$ & $\frac{3 \pi}{8}$ & $\frac{7 \pi}{16}$ & $\pi / 2$ \\
\hline 1.550883197 & & 1.482881951 & 1.243027662 & 0.7711704511 & 0 \\
\hline
\end{tabular}

Using Romberg Approximation

$$
h=\frac{\pi / 2-0}{8}=\frac{\pi}{16} ; f(x)=e^{x} \cos x
$$

Table 12. Values of $x$ against $f(x)=e^{x} \cos x$ for Rombeg Integration with 8 subdivisions.

Using trapezoidal rule with 1 subdivision.

$$
\begin{gathered}
h=\frac{\pi / 2-0}{1}=\pi / 2 \\
T_{1}=\int_{0}^{\frac{\pi}{2}} e^{x} \cos x d x=\frac{1}{2} h\left(f_{0}+f_{8}\right) \\
\Rightarrow T_{1}=\frac{1}{2} \cdot \frac{\pi}{2}(1+0) \\
=0.7853981634
\end{gathered}
$$

Using trapezoidal rule with 2 subdivisions.

$$
h=\frac{\pi / 2-0}{2}=\pi / 4
$$




$$
\begin{aligned}
& T_{2}=\int_{0}^{\pi / 2} e^{x} \cos x d x=\frac{1}{2} h\left(f_{0}+2 f_{4}+f_{8}\right) \\
& \Rightarrow T_{2}=\frac{1}{2}(\pi / 4)(1+2(1.550883197)+0) \\
& =1.610759896
\end{aligned}
$$

Using trapezoidal rule with 4 subdivisions.

$$
\begin{gathered}
h=\frac{\pi / 2-0}{4}=\pi / 8 \\
T_{4}=\int_{0}^{\pi / 2} e^{x} \cos x d x=\frac{1}{2} h\left(f_{0}+2\left(f_{2}+f_{4}+f_{6}\right)+f_{8}\right) \\
\Rightarrow T_{4}=\frac{1}{2}(\pi / 8)\left(1+2\left(\begin{array}{l}
1.368240338+1.550883197 \\
+1.243027662
\end{array}\right)+.0\right) \\
=1.830822494
\end{gathered}
$$

Using trapezoidal rule with 8 subdivisions.

$$
\begin{aligned}
& h=\frac{\pi / 2-0}{8}=\pi / 16 \\
& T_{8}=\int_{0}^{\pi / 2} e^{x} \cos x d x=\frac{1}{2} h\left(f_{0}+2\left(\begin{array}{l}
f_{1}+f_{2}+f_{3} \\
+f_{4}+f_{5}+f_{6} \\
+f_{7}
\end{array}\right)+f_{8}\right) \\
& \Rightarrow T_{8}=\frac{1}{2}\left(\pi / 16\left(1+2\left(\begin{array}{l}
1.19356881+1.368240338 \\
+1.498535197+1.550883197+ \\
1.482881951+ \\
1.243027662+0.7711704511
\end{array}\right)\right)+0\right) \\
& =1.886586787
\end{aligned}
$$

Now, using Romberg approximation, we apply the iterative formula below to obtain the Romberg extrapolates as shown below

$$
T_{2 N, i+1}=\frac{4 T_{2 N, i}-T_{N, i}}{3}
$$

Table 13. Values of $x$ against $f(x)=e^{x} \cos x$ for Rombeg Iterative formula with 8 subdivisions.

\begin{tabular}{lllll}
\hline $\boldsymbol{N}$ & $\boldsymbol{T}_{N}$ & $\boldsymbol{T}_{2 N, 1}$ & $\boldsymbol{T}_{2 N, 2}$ & $\boldsymbol{T}_{2 N, 3}$ \\
\hline 1 & 0.7853981634 & 1.885880474 & & \\
2 & 1.610759896 & 1.904176693 & 1.910275433 & 1.903918343 \\
4 & 1.830822494 & 1.905174885 & 1.905507615 & \\
8 & 1.886586787 & & \\
\hline
\end{tabular}

So the Romberg approximation gives $I=\int_{0}^{\pi / 2} e^{x} \cos x d x=1.903918343$ 


$$
\begin{aligned}
& \left|\varepsilon_{t}\right|=\left|\frac{1.90523869-1.903918343}{1.90523869}\right| \times 100 \% \\
& \approx 6.930089 \times 10^{-2} \mathrm{o} / \mathrm{o}
\end{aligned}
$$

Using Boole's rule with $n=4$

$$
\begin{gathered}
\mathrm{h}=\frac{\pi / 2}{4}=\frac{\pi}{8} \\
I=\frac{2 h}{45}\left(7 y_{0}+32 y_{1}+12 y_{2}+32 y_{3}+7 y_{4}\right) \\
I=\frac{2 \times(0.392857)}{45}\left(\begin{array}{l}
7 \times 1+32 \times 1.368367+12 \times 1.550883 \\
+32 \times 1.242193+7 \times-0.00304
\end{array}\right) \\
I=1.905396 \\
\left|\varepsilon_{\mathrm{t}}\right|=\left|\frac{1.90523869-1.905396}{1.90523869}\right| \times 100 \% \\
\approx 8.26 \times 10^{-3} \%
\end{gathered}
$$

Using Boole's rule with $n=8$

$$
\begin{gathered}
\mathrm{h}=\frac{\pi / 2}{8}=\frac{\pi}{16} \\
I=\frac{2 h}{45}\left[7 y_{0}+32\left(y_{1}+y_{3}+y_{5}+y_{7}\right)+12\left(y_{2}+y_{6}\right)+14 y_{4}+7 y_{8}\right] \\
I=\frac{2 \times(0.196429)}{45}\left(\begin{array}{l}
7 \times 1+32(1.193644+1.498653+1.482591 \\
+0.769451)+12(1.368367+1.242193) \\
+(14 \times 1.550883)+(7 \times-0.00304)
\end{array}\right) I=1.90524 \\
\left.\mid \begin{array}{l}
\varepsilon_{\mathrm{t}}|=| \frac{1.90523869-1.90524}{1.90523869} \mid \\
\approx 9.38631 \times 10^{-5} \%
\end{array}\right) \times 100 \%
\end{gathered}
$$

Using weddles 's rule with $n=6$

$$
\begin{gathered}
\mathrm{h}=\frac{\pi / 2}{6}=\frac{\pi}{12} \\
I=\frac{3 h}{10}\left[y_{0}+5 y_{1}+y_{2}+6 y_{3}+y_{4}+5 y_{5}+y_{6}\right] \\
I=\frac{3 \times 0.261905}{10}\left[\begin{array}{l}
1+5 \times 1.255091+1.462061+6 \times 1.550883 \\
+1.424387+5 \times 0.956886+-0.00304
\end{array}\right] \\
I=1.905246
\end{gathered}
$$




$$
\begin{aligned}
& \left|\varepsilon_{\mathrm{t}}\right|=\left|\frac{1.90523869-1.905246}{1.90523869}\right| \times 100 \% \\
& \approx 4.0 \times 10^{-4} \%
\end{aligned}
$$

Using the 2-point DIAF

$\mathrm{N}=2 ; h=\frac{\pi / 2-0}{4(2)}=\frac{\pi}{16} ; f(x)=e^{x} \cos x ; w(x)=1$

So that, $I=\int_{0}^{\pi / 2} e^{x} \cos x d x=\frac{2(\pi / 16)}{45} \sum_{k=0}^{8} \lambda_{k} f\left(\frac{\pi k}{16}\right)$

Then for the 2-point DIAF, the weights are given by $\lambda_{k}=\left\{\begin{array}{l}7, \text { if }(k=\{0,8\}) \\ y, \text { otherwise }\end{array}\right.$

where $y=\left[32+(-1)^{k-1}\left(\frac{\left(2^{k}+(-2)^{k}\right)}{\left\{\frac{r}{2} \frac{2^{k-1}}{5}+\left(1-\frac{r}{2}\right) \frac{2^{k}}{9}\right\}}\right]\right]$

and $r=k \bmod 4$

$$
\begin{aligned}
& \lambda_{0}=7 \\
& \lambda_{1}=\left[32+(-1)^{1-1}\left(\frac{\left(2^{1}+(-2)^{1}\right)}{\left.\left\{\frac{1}{2} \frac{2^{1-1}}{5}+\left(1-\frac{1}{2}\right) \frac{2^{1}}{9}\right\}\right)}\right], \lambda_{2}=\left[32+(-1)^{2-1}\left(\frac{\left(2^{2}+(-2)^{2}\right)}{\left\{\frac{2}{2} \frac{2^{2-1}}{5}+\left(1-\frac{2}{2}\right) \frac{2^{2}}{9}\right\}}\right)\right]\right. \\
& =32+\left(\frac{0}{\frac{1}{2}\left(\frac{2^{0}}{5}\right)}\right)=32 \quad=32-\left(\frac{2^{3}}{\left(\frac{2}{5}\right)}\right)=12 \\
& \lambda_{3}=\left[32+(-1)^{3-1}\left(\frac{\left(2^{3}+(-2)^{3}\right)}{\left\{\frac{3}{2} \frac{2^{3-1}}{5}+\left(1-\frac{3}{2}\right) \frac{2^{3}}{9}\right\}}\right)\right] \\
& =32+\left(\frac{0}{\frac{3}{2} \frac{2^{3-1}}{5}+\left(1-\frac{3}{2}\right) \frac{2^{3}}{9}}\right)=32
\end{aligned}
$$




$$
\begin{aligned}
\lambda_{4} & =\left[32+(-1)^{4-1}\left(\frac{\left(2^{4}+(-2)^{4}\right)}{\left\{\frac{0}{2} \frac{2^{4-1}}{5}+\left(1-\frac{0}{2}\right) \frac{2^{4}}{9}\right\}}\right)\right] \\
& =32-\left(\frac{2^{5}}{\left(\frac{2^{4}}{9}\right)}\right)=14
\end{aligned}
$$

Similarly, it follows that on substitution:

$$
\lambda_{5}=32, \lambda_{6}=12, \lambda_{7}=32, \lambda_{8}=7
$$

Thus,

$$
\begin{aligned}
& \Rightarrow I=\frac{2(\pi / 16)}{45}\left\{\begin{array}{l}
7\left(e^{0} \cos (0)\right)+32\left(e^{\pi / 16} \cos (\pi / 16)\right) \\
+12\left(e^{\pi / 8} \cos (\pi / 8)\right) \\
+32\left(e^{3 \pi / 16} \cos (3 \pi / 16)\right) \\
+14\left(e^{\pi / 4} \cos (\pi / 4)\right) \\
+32\left(e^{5 \pi / 16} \cos (5 \pi / 16)\right) \\
+12\left(e^{3 \pi / 8} \cos (3 \pi / 8)\right) \\
+32\left(e^{7 \pi / 16} \cos (7 \pi / 16)\right) \\
+7\left(e^{\pi / 2} \cos (\pi / 2)\right)
\end{array}\right\} \\
& \Rightarrow I=\frac{\pi}{360}\left(\begin{array}{l}
7(1)+32(1.19356881) \\
+12(1.368240338) \\
+32(1.498535197) \\
+14(1.550883197) \\
+32(1.482881951) \\
+12(1.243027662) \\
+32(0.7711704511)+7(0)
\end{array}\right) \\
& =1.905241431 \\
& \left|\varepsilon_{t}\right|=\left|\frac{1.90523869-1.905241431}{1.90523869}\right| \times 100 \% \\
& \approx 1.43843 \times 10^{-4} \%
\end{aligned}
$$

Table 14. Summary of the Test Results.

\begin{tabular}{llll}
\hline Integral & Formula & Integral Value & Error \\
\hline 1 & 2-Point DIAF & 0.693147901 & $1.0394 \times 10^{-4} \%$ \\
$\int_{0}^{1} \frac{1}{x+1} d x$ & Gauss-Chebyshev 3 Point & 0.729864959 & $5.30 \%$ \\
0 & Gauss-Legendre 3 Point & 0.693121693 & $3.6771 \times 10^{-3} \%$ \\
\hline
\end{tabular}




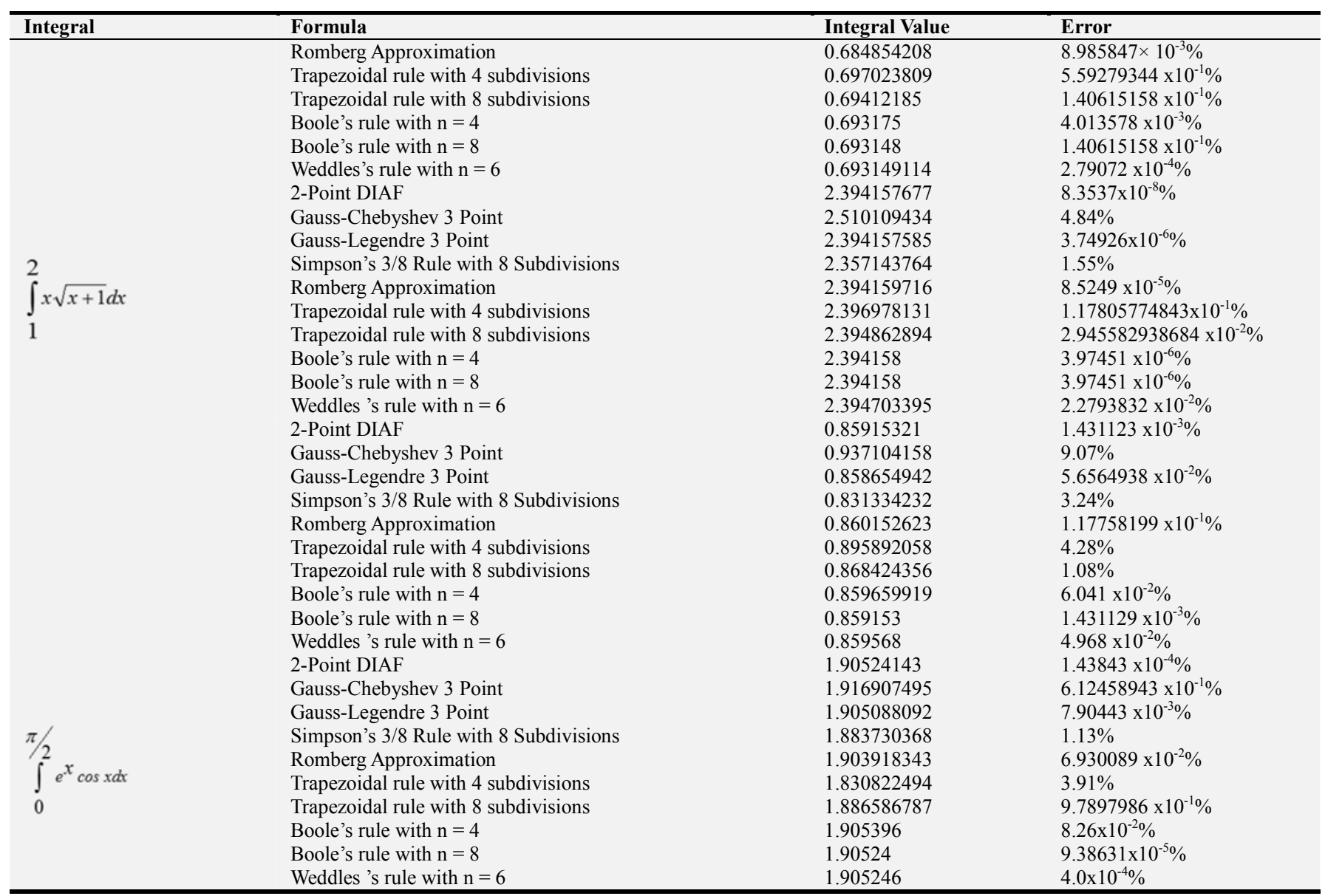

\section{Precision and Stability}

Definition 4.1: The degree of accuracy or precision of a quadrature formula is the largest positive integer $\mathrm{n}$ such that the formula is exact for $x^{k}$, for each $k=0,1,2, \ldots \ldots, n$.

Trapezoidal rule has degree of accuracy one while Simpson's rule has degree of accuracy three.

Remark: The degree of precision of a quadrature formula is $\mathrm{n}$ if and only if the error is zero for all polynomials of degree $k=0,1, \ldots . ., n$, but is NOT zero for some polynomial of degree $n+1$.

Remark: $N$ is even, degree of precision is $N+1 . N$ is odd, degree of precision is $N$

For the N-point Definite Integral Approximation Formula (N-point DIAF), if $N=2$ then the degree of precision is three while if $N=3$ then the degree of precision is three.

In addition to having a stable problem, i.e., a problem for which small changes in the initial conditions elicit only small changes in the solution, there are two basic notions of numerical stability. The first notion of stability is concerned with the behaviour of the numerical solution for a fixed value $t>0$ as $h \rightarrow 0$.

Definition 4.2 A numerical integration method is zero stable if small perturbations in the initial conditions do not cause the numerical approximation to diverge away from the true solution provided the true solution of the initial value problem is bounded.

For a consistent s-step method one can show that the notion of stability and the fact that its characteristic polynomial $\rho$ satisfies the root condition are equivalent. Therefore, as mentioned earlier, for an s-step method we have convergence $\Leftrightarrow$ consistent \& stable.

This concept of stability also plays an important role in determining the global truncation error. In fact, for a convergent (consistent and stable) method the local truncation errors add up as expected, i.e., a convergent s-step method with $\mathrm{O}\left(h^{p+1}\right)$ local truncation error has a global error of order $\mathrm{O}\left(h^{p}\right)$.

The N-point Definite Integral Approximation Formula (Npoint DIAF) is stable order $p=0$ and unstable at $p=N+1$.

Thus, N-DIAF method is only conditionally stable, i.e., the step size has to be chosen sufficiently small to ensure stability.

\section{Conclusion}

From the above tests, it is evident that the 2-Point DIAF has the lowest relative error compared to other numerical integration techniques. As such it gives a better approximate solution to the integration i.e. it easily converges to the exact value $[10]$ and is more stable. 


\section{Recommendations}

A comparison with other numerical integration methods, such as according to [7] Gauss-Legendre 3 point formula, Gauss-Chebyshev 3 point formula, Simpson's Rule, and Romberg method, demonstrated that the N-point DIAF gives a better precision, as depicted by the test problem above. The error involved in using the N-point DIAF is the least. It is therefore recommended that the Npoint DIAF should be implemented by Scientists and Engineers to help in approximating definite integrals especially those that cannot be evaluated using analytical techniques.

\section{Acknowledgements}

My thanks to the many authors of books, papers, and articles as well as a new generation of contributors to electronic media (the World Wide Web) who have provided us with additional insight and ideas. Special thanks and acknowledgement also go to my lecturer, Mr. Nicholas M. Muthama of Taita Taveta University, for having imparted me with the necessary knowledge and skills to be able to apply while discovering this formula and also for his insightful, constructive, and valuable comments. I also wish to thank Mr. Nicholas Mutothya for his contribution in an attempt to compare the N-point DIAF with the neighborhood method used in Regression Analysis (i.e. the k-Nearest Neighbors Regression algorithm). Special thanks go to Prof. Hamadi Boga, the Vice Chancellor Taita Taveta University for his support towards completion of this research.

\section{References}

[1] Burden, R. L. and Faires, J. D., Numerical Analysis, $9^{\text {th }}$ ed., Brooks/Cole, Pacific Grove, 2011. pp 193-256.

[2] C. -H. Yu, Solving some integrals with Maple, International Journal of Research in Aeronautical and Mechanical Engineering, Vol. 1, Issue. 3, pp. 29-35, 2013.

[3] Dukkipati, R. V., Numerical Methods, New Age International Publishers (P) Ltd., New Delhi, India, 2010. Pp 237-263.

[4] Gordon K. Smyth (1998), Numerical Integration, Encyclopedia of Biostatistics (ISBN 0471 975761), John Wiley \& Sons, Ltd, Chichester.

[5] Gupta, C. B and Malik, A. K. (2009), Advanced Mathematics, (New Age International Publishers) Pp 90-101.

[6] Jain, M. K., S. R. K. Iyengar., and R. K. Jain, Numerical Methods for Scientific and Engineering Computation, Sixth Edition, New Age International Publishers, (Formerly Wiley Eastern Limited), New Delhi, 2008. Pp 128-177.

[7] Jain, M. K., S. R. K. Iyengar., and R. K. Jain, Numerical Methods for Scientific and Engineering Computation, Sixth Edition, New Age International Publishers, (Formerly Wiley Eastern Limited), New Delhi, 2012. Pp 128-177.

[8] K. E. Atkinson, An Introduction to Numerical Analysis, John Wiley and Sons, New York, NY, USA, Second Edition, 1989.

[9] Rao, G. S. (2006), Numerical Analysis, Revised Third Edition (New Age International (P) Limited Publishers).

[10] T. Ramachandran, R. Parimala (2015), Open Newton - Cotes Quadrature With Midpoint Derivative For Integration Of Algebraic Functions, International Journal of Research in Engineering and Technology, Volume: 04 Issue: 10. 